\title{
Commenter Galien et Hippocrate à la Renaissance ou comment Brasavola met à mal le régime grec
}

ANTOINE PIETROBELLI

Université de Reims Champagne-Ardenne

This paper aims to draw attention on a commentary written by the famous Antonio Musa Brasavola upon the Galenic Commentary on Regimen in Acute Diseases. Published in 1546 by this disciple of Leoniceno and Manardi, this work is a very brilliant illustration of the Ferrara's medical Hellenism. Returning to Galenic and Hippocratic Greek texts, Brasavola shows a huge classical scholarship in his interpretation. But surprisingly for a follower of the Hellenists, Brasavola mentions also many Arabic writers to make them converse with Greek authorities. Confronting the medical tradition of the past with his own experience as a practitioner, Brasavola seems to cast doubt on some aspects of the Greek regimen in acute diseases, opening the way to significant changes in the theories of digestion and fever.

$\mathrm{L}^{2}$ a littérature médicale offre sans doute un bel échantillon, si l'on veut observer à la loupe le processus de réappropriation et de transformation du savoir des anciens qui s'est opéré durant la Renaissance. Pour aller vite, on pourrait retracer à grands traits cette évolution : la redécouverte de la médecine grecque dans sa langue originale, balayant l'héritage scolastique des traductions arabo-latines, occasionna une révolution scientifique qui eut pour levier les découvertes anatomiques exposées par André Vésale dans son De humani corporis fabrica (1543) ou physiologiques publiées par William Harvey dans son De motu cordis (1628). Pourtant, cette vision schématique et raccourcie a été nuancée, voire déconstruite depuis une vingtaine d'années.

Tout d'abord, le phénomène européen de réception des médecins grecs à la Renaissance fut long, lent et complexe $e^{1}$. Le renouveau médical débute en Italie, dès les années 1480 , avec l'apparition des premières traductions humanistes ${ }^{2}$. Durant la décennie suivante, ce mouvement s'intensifie sous l'égide de Niccolò 
Leoniceno (1428-1524) qui remet en cause un enseignement et une pratique de la médecine fondés sur les traductions arabo-latines. Leoniceno condamne ces traductions médiévales, jugées trop obscures et barbares. Il en appelle à un retour aux originaux grecs pour purifier les connaissances médicales. Cette rhétorique, qui s'en prend au passage au legs des médecins arabes ${ }^{3}$, ouvre la voie à un humanisme philologique et devient un leitmotiv dans les préfaces des premiers éditeurs des textes médicaux grecs. Une seconde étape, à partir des impressions aldines des œuvres de Galien (1525-1526) et d'Hippocrate (1526), voit le plein essor du mouvement de l'hellénisme médical caractérisé par une érudition textuelle très poussée ${ }^{4}$. Après 1550-1555, l'approche philologique décline peu à peu et les médecins, pour l'avoir pleinement assimilé, s'affranchissent progressivement du modèle galénique ${ }^{5}$.

Il faut en outre souligner un point paradoxal : étudier l'impact de la redécouverte des médecins grecs et l'influence de leur médecine à la Renaissance implique de lire des textes écrits en latin ${ }^{6}$. Sans parler de l'étape pré-éditoriale, où la connaissance des manuscrits grecs se limite à quelques érudits collectionneurs, l'accès aux sources grecques reste un épiphénomène, même après la parution des Aldines de Galien et d'Hippocrate, en raison du coût des volumes imprimés et de la faible proportion d'hellénistes parmi les médecins et les praticiens. La diffusion de la médecine grecque s'est avant tout effectuée par le biais des nouvelles traductions latines humanistes réalisées après la publication des éditions principes. Et les progrès de la science médicale ont été véhiculés en langue latine ${ }^{7}$.

Durant les années 1540, qui consacrent le triomphe de l'hellénisme médical, le médecin Antonio Musa Brasavola (1500-1555) entreprend de commenter en latin le commentaire de Galien sur le Régime des maladies aiguës d'Hippocrate. C’est en 1546 que paraît à Venise chez Jérôme Scot son ouvrage In libros de ratione victus in morbis acutis, Hippocratis et Galieni commentaria et annotationes (= Commentaires et annotations). Le mot à mot de ce commentaire d'Hippocrate et de Galien permet de suivre pas à pas le dialogue qu'établit cet érudit de la Renaissance avec les modèles du passé.

Antonio Musa Brasavola ${ }^{8}$ est l'un des médecins les plus renommés de son temps. C'est à Ferrare qu'il fit ses études humanistes et poursuivit sa carrière de professeur. Disciple de Leoniceno - dont il fit la biographie ${ }^{9}$ - et de Giovanni Manardi (1462-1536), il enseigna d'abord la logique et la philosophie naturelle, puis obtint la chaire de médecine théorique en 1537 et celle de médecine 
pratique en 1541. Entré comme médecin au service d'Hercule II d'Este, fils d'Alphonse Ir duc de Ferrare, dès 1521, il offrit également ses consultations aux plus puissants de l'époque : le pape Paul III, les rois François $\mathrm{I}^{\text {er } 10}$ et Henri VIII, et l'empereur Charles Quint sont à compter parmi ses nombreux patients.

De son œuvre qui embrasse bien d'autres champs que la médecine ${ }^{11}$, la postérité a essentiellement retenu deux apports majeurs. Brasavola est tout d'abord connu pour être l'auteur d'un Index ${ }^{12}$ monumental qui accompagne les éditions juntines du Galien latin. Cet Index de 1094 pages impressionne aujourd'hui, car il fut constitué minutieusement en un temps où n'existaient pas les ressources électroniques. Irremplaçable pour des générations de médecins ${ }^{13}$, cet Index fournit une belle illustration de l'érudition qui se déploie sur le nouveau Galien des traductions humanistes. D’autre part, Brasavola se pose en digne successeur de Leoniceno et de Manardi par ses progrès dans les domaines de la botanique et de la pharmacologie. Dans la lignée de ses prédécesseurs, Brasavola poursuit l'identification des espèces végétales et de leurs propriétés thérapeutiques en collectant les noms de plantes dans les langues anciennes et modernes pour les appliquer aux spécimens prélevés lors de ses herborisations ${ }^{14}$. L'Index de Brasavola et son importante contribution à la botanique donnent une idée de l'envergure de son érudition.

Cette étude sur les Commentaires et annotations de Brasavola aux textes de Galien et d'Hippocrate sur le régime des maladies aiguës porte principalement sur la première partie de l'ouvrage ${ }^{15}$, mais elle se propose d'apporter quelques éclairages nouveaux sur les pratiques textuelles et médicales du médecin de Ferrare.

De manière générale, les éditeurs modernes d'Hippocrate et de Galien ont accordé peu d'intérêt aux commentaires des médecins de la Renaissance, car ces écrits n’apportent rien à l'établissement du texte. Pourtant, du point de vue de l'histoire de la médecine, cette production exégétique doit être prise en compte pour comprendre la fortune et la réception du galénisme au XVI ${ }^{\mathrm{e}}$ siècle ${ }^{16}$. Depuis le XIII siècle, le Régime des maladies aiguës d'Hippocrate avait été abondamment commenté dans les universités de médecine occidentales. Mais les Commentaires et annotations de Brasavola, entre tradition et innovation, revisitent un standard de la littérature médicale médiévale, en mobilisant l'imposante érudition classique d'un humaniste. Cette nouvelle lecture est révélatrice d'une culture propre autant que d'une pratique médicale spécifique. 
Formé à Ferrare par Leoniceno et Manardi, Brasavola appartient au clan des médecins hellénistes. Connaissant le grec et rompu aux méthodes philologiques, il travaille, comme ses maîtres, à purger la science médicale des altérations et des imprécisions terminologiques dues aux traductions arabolatines. Leoniceno ${ }^{17}$ et surtout Manardi ne ménagent pas leurs attaques contre la médecine arabe : ce dernier critique violemment l'autorité d'Avicenne dans ses Epistulae medicinales ${ }^{18}$. Pourtant, quand on lit les Commentaires et annotations de Brasavola, on est frappé par le grand nombre de sources arabes qui sont convoquées pour l'interprétation du texte. Il y a peut-être dans ce dialogue entre les sources classiques et arabes un trait original de Brasavola par rapport à ses anciens maîtres.

Un autre intérêt de ce commentaire est lié à son thème diététique. Les professeurs de médecine de la Renaissance qui reconduisaient d’année en année l'étude des grands textes scolaires du Moyen Âge, étaient encore pleinement tributaires des catégories antiques. Dans l'Antiquité, la médecine thérapeutique était divisée en trois branches : la chirurgie, la diététique et la pharmacologie ${ }^{19}$. Les traités pratiques s'organisaient suivant ces trois pôles thérapeutiques. Grâce aux études des historiens de la médecine, on perçoit aujourd'hui assez bien comment les médecins de la Renaissance ont fait évoluer la discipline de la chirurgie $^{20}$. Pour la pharmacologie, le renouveau est également palpable. La révision de la pharmacopée antique donne naissance aux sciences modernes de la botanique ${ }^{21}$, comme en témoigne l'école de Ferrare, ou de la minéralogie, avec les recherches de l'un des éditeurs des aldines d'Hippocrate et de Galien, le saxon Georg Agricola ${ }^{22}$ (1494-1555). Il est clair que pour la chirurgie et la pharmacologie, les hommes de la Renaissance ont transformé une organisation ancienne des savoirs pour construire des catégories appelées à devenir des disciplines scientifiques modernes. Pour la diététique en revanche, le lien est moins évident. Il semble que cette spécialité antique s'efface du champ de la médecine à la Renaissance et qu'elle ne passe pas le cap épistémologique de la modernité. Pourtant, ainsi que le rappelle Brasavola dans son introduction, la définition du régime (diaita) antique n'englobe pas seulement les aliments et les boissons, elle intègre aussi d'autres paramètres tels le repos, l'exercice, les bains, l'activité sexuelle, le sommeil et la veille et tout ce qui engage le corps ${ }^{23}$. Un disciple célèbre de Brasavola, Gabriele Falloppio (1523-1562), considérait son maitre comme un anathomicorum atque herbariorum coryphaeus, mais quel sort fit le médecin de Ferrare à la troisième voie de la médecine antique? 
Comment a-t-il discuté, en homme de son temps, du régime prescrit par les anciens?

\section{Relire les classiques}

Le projet exégétique de Brasavola peut dénoter une forme de conservatisme. De fait, à partir de la traduction en langue latine du Régime des maladies aiguës (= RMA) d'Hippocrate par Constantin l'Africain, puis du Commentaire au Régime des maladies aiguës (= CRMA) de Galien par Gérard de Crémone, on commenta le traité hippocratique dans les universités de médecine européennes. En mettant cette œuvre au programme, deux options s'offraient alors aux professeurs : expliquer le texte hippocratique grâce au commentaire de Galien, ou bien l'interpréter en produisant leur propre exégèse. C'est ainsi qu'un grand nombre de maîtres médiévaux ${ }^{24}$ ont rédigé, comme Galien, un commentaire du Régime des maladies aiguës hippocratique. Mais le commentaire galénique connut également un grand succès. Le CRMA de Galien fut inscrit, à partir de la seconde moitié du XIII e siècle, au programme des universités de médecine à Padoue, Bologne, Paris et Oxford, pour ne citer que les plus célèbres. Le commentaire de Galien faisait partie de recueils-types dénommés Ars commentata ${ }^{25}$ et Articella $^{26}$ que tout étudiant en médecine se devait d'acquérir afin daccomplir son cursus universitaire. En choisissant ce texte, Brasavola se conforme donc à une tradition universitaire, il reproduit scrupuleusement l'enseignement de ses maîtres ${ }^{27}$. À ce propos, il est significatif quavant d'entreprendre ses Commentaires et annotations sur le CRMA, Brasavola ait produit un ouvrage exégétique sur le Commentaire aux Aphorismes ${ }^{28}$ et un autre sur le Commentaire au Pronostic ${ }^{29}$, s'attachant ainsi à l'interprétation de deux commentaires hippocratiques de Galien qui précédaient traditionnellement le CRMA dans les recueils médiévaux de l'Ars commentata et de l'Articella.

Par rapport à cette lignée exégétique séculaire, l’entreprise de Brasavola se distingue pourtant de deux façons : d'une part, il fonde son interprétation sur un texte latin directement traduit du grec sans plus passer par l'intermédiaire arabe. Même quand il la modifie, il prend pour texte de base la traduction latine de Jean Vassé, effectuée à partir du texte grec de l'Aldine et publiée pour la première fois en 1531 chez l'imprimeur parisien Simon de Colines. Ses renvois aux autres ouvrages de Galien montrent que Brasavola les connait également 
par le biais des novae translationes. D’autre part, Brasavola explique autant les lemmes d'Hippocrate que le commentaire de Galien : il produit un commentaire du commentaire, exercice laborieux et périlleux auquel ne s'étaient pas risqués les maîtres médiévaux.

\section{Un ouvrage scolaire}

Les Commentaires et annotations forment un volumineux in-folio. Le livre est composé de quatre tomes, soit un par livre et compte 592 pages $^{30}$. L'édition imprimée adopte une présentation uniforme. Le texte est disposé sur deux colonnes. Chaque lemme d'Hippocrate - précédé du nom Hippocrates en minuscule et de son numéro d'ordre introduit par le terme sectio - est écrit en gras, dans une police de plus gros module; puis vient un commentaire de Brasavola du texte hippocratique, puis le commentaire de Galien, puis un autre commentaire de Brasavola du commentaire galénique. Sur la page, les deux exégètes sont typographiquement mis sur le même plan, puisque leur texte est écrit dans une police de même taille et annoncé par leur nom en majuscule soit dans le corps du texte, soit dans la marge. Dans ce commentaire-gigogne de Brasavola, une des constantes est de proposer la plupart du temps un commentaire plus long pour Hippocrate que pour Galien, bien que le lemme soit par essence toujours plus court que le commentaire galénique. Autre constante : Brasavola est toujours plus prolixe qu'Hippocrate et que Galien. La progression concentrique donne un écho au moindre mot hippocratique et allonge considérablement le texte. Cet exercice de commentaire emboîté est pourtant délicat : bien souvent, le premier commentaire de Brasavola coupe, pour ainsi dire, l'herbe sous le pied de Galien en reprenant les grandes lignes de ce que sera le commentaire galénique. La progression malhabile et compliquée peut parfois paraître redondante. Pour comprendre cette rhétorique de la répétition, il faut replacer l’ouvre dans le contexte de sa production.

Tout d'abord la forme du commentaire hippocratique de Galien présuppose la situation d'énonciation suivante : après avoir lu le texte d'Hippocrate, Galien propose à ses disciples une exégèse des mots obscurs et des notions médicales requises $^{31}$. La rédaction du commentaire suppose l'expérience orale d'une leçon, où le maître commente le texte qui fait autorité devant ses disciples. Les marques d’oralité et de dialogue qui foisonnent dans le commentaire galénique ne 
laissent aucun doute sur cette situation énonciative originelle. On peut ensuite établir que le succès que rencontrèrent certains commentaires hippocratiques de Galien au Moyen Âge tient au fait que la cellule de base maître-disciple est demeurée inchangée à travers les âges. Les commentaires de Galien sont donc restés particulièrement adaptés à l'enseignement de la médecine. Dans le cas de Brasavola, il est révélateur que la rédaction de ses annotations sur les commentaires hippocratiques de Galien coïncide avec le moment où il obtient la chaire de médecine pratique à l'université de Ferrare ${ }^{32}$. Son texte conserve lui aussi les traces d'un contexte scolaire. En effet, ces commentaires sont souvent structurés en fonction d'un dialogue sous-jacent. Par exemple au premier paragraphe du f. 78 , col. a, alternent : inquies... respondemus... at dices... respondemus... at dices... respondemus... dices uero... respondemus... forte etiam dubitabis... respondemus ... petes... respondemus. Cette succession de questions-réponses avec un interlocuteur fictif qui scande tout du long les Commentaires et annotations rappelle assurément la forme dialogique que Brasavola affectionne dans plusieurs de ses traités ${ }^{33}$, mais cette alternance de la première et de la deuxième personne évoque aussi le dialogue qui s'institue dans une salle de cours entre le maître et ses disciples ${ }^{34}$. Pédagogue, Brasavola fait montre d'une grande clarté d'exposition en hiérarchisant systématiquement ses remarques par les adverbes : primo... secundo... tertio... quarto... quinto... etc. La rédaction des Commentaires et annotations est donc le résultat d'un cours ou d'un séminaire que donnait Brasavola à Ferrare. Dans son prologue, il explique qu'il a commencé à préparer son livre dès 1542, à l'occasion d'un cours dispensé aux jeunes gens de Ferrare :

Nullus uero est qui de ratione uictus in morbis acutis pertractet praeter hunc, quem hoc anno.1542. Ferrariensi iuuentuti exponendum suscepimus. (prol. p. 2, col. a, l. 68-col. b, 1. 3)

[Il n'existe aucun ouvrage qui examine à fond le régime dans les maladies aiguës, hormis celui que, durant cette année 1542, j’ai entrepris d'exposer à la jeunesse de Ferrare.] 


\section{Une approche philologique}

L'école de Ferrare initiée par Leoniceno est caractérisée, selon V. Nutton, par son acuité philologique (" concentration on the meaning of individual words») Dans l'édition latine de Brasavola, on trouve des mots et même des phrases notés en alphabet grec. Brasavola connaît le grec, il le traduit, il est capable de discuter des variantes textuelles. Ainsi, après le texte des lemmes donné en latin dans la traduction de Jean Vassé légèrement révisée, Brasavola, quand il commente Hippocrate, reprend chaque phrase du lemme en grec - dans le texte de l'Aldine de Galien, à quelques menus détails près - pour les traduire et les commenter séparément. Ce travail sur le grec ne concerne pas uniquement les lemmes hippocratiques. Des mots grecs émaillent aussi les commentaires du Ferrarais sur le texte galénique. Parfois, quand l'Aldine lui semble fautive, Brasavola reprend le texte grec de Galien pour le corriger. En I 15 G (= sectio $\mathrm{XVI})^{36}$, quand Galien expose que pour la mantique, les prédictions dépendent du genre de la personne, il définit que les femmes, les invertis et ceux qui ont une activité féminine sont à ranger dans la même catégorie. Il cite alors un exemple d'hommes qui, ayant une activité de femme, sont régis par les mêmes lois divinatoires que les femmes. L'exemple qu'il donne est celui du « lainier » (غ́pıoupyóv), mais l'Aldine commet une faute en donnant le mot « sacrificateur »

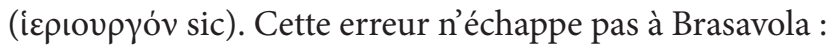

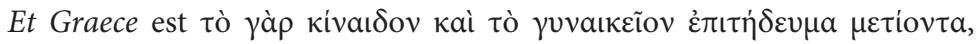

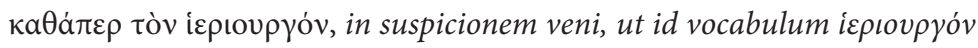

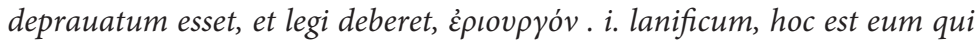
lanas aptat ...

(p. 22, col. a, 1. 59-63).

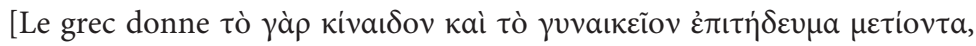

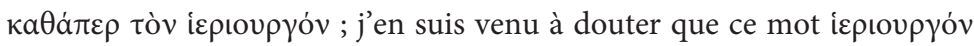

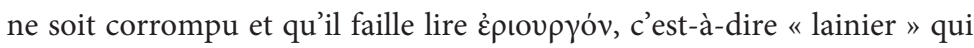
désigne celui qui travaille la laine.]

Brasavola montre aussi qu'il connaît la leçon d'un manuscrit grec quand il écrit : 
Scito tamen hanc partem, quod zeae nullo inest pacto, in omnibus impressis

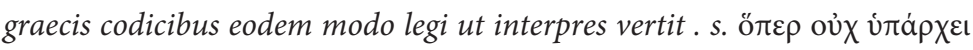

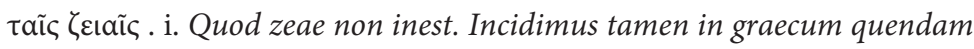

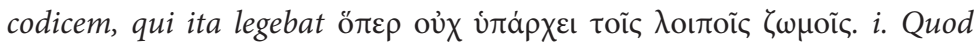
reliquis iuribus non inest, si haec uera sit lectio, intelligenda est de iuribus ex chondro, ex farre, ex zea et similibus aliis (p. 37, col. a, 1. 8-15).

[Sache pourtant que ce passage, « qui n'existe nullement dans le zea (amidonnier) » se lit dans toutes les éditions imprimées grecques comme

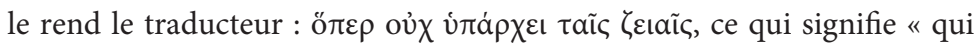
n'existe pas dans le zea (amidonnier) ». Nous sommes toutefois tombé

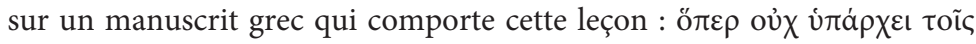
$\lambda$ oıтoĩ $\zeta \omega \mu$ oĩ , « ce qui n'existe pas dans les autres soupes ». Si cette leçon est la bonne, il faut comprendre ces soupes comme étant celles à base de gruau, de blé, d'amidonnier et d'autres céréales de cette sorte.]

Pour ce passage, Brasavola a effectivement l'intuition de la leçon qu'il faut éditer contre les éditions imprimées du XVI siècle. Il semble en outre que Brasavola ait pu collationner plusieurs manuscrits grecs - ou du moins des éditions imprimées annotées à partir de manuscrits - avant d’entreprendre son commentaire ${ }^{37}$.

Le médecin italien est donc attentif, quand il utilise la traduction de Jean Vassé, au texte grec de l'Aldine dont elle découle et il est à même de corriger le texte-source par des conjectures ou par des leçons empruntées à d’autres manuscrits ${ }^{38}$. Brasavola corrige aussi parfois les inexactitudes de la traduction

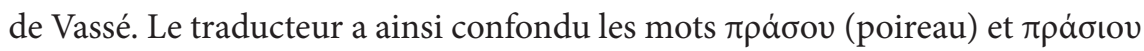
(marrube) et donc deux espèces végétales bien distinctes. Il note :

At unus error est non dissimulandus in hoc interprete (...). Aldinus codex

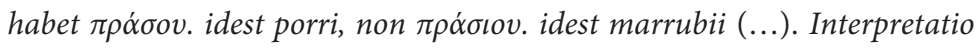
antiqua melius vertit (p. 70, col. b, 1. 56-p. 71, col. a, 1. 5).

[Il est une erreur qu'on ne saurait cacher chez ce traducteur (...). L'Aldine

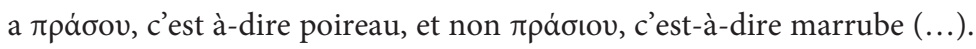
La traduction latine ancienne est meilleure... ] 
Pour montrer l'erreur de Jean Vassé, il a recours ici, entre autres, au témoignage de l'ancienne traduction arabo-latine de Gérard de Crémone, ce qui montre qu'il disposait de cet outil pour la rédaction des Commentaires et annotations. On pourrait multiplier les exemples pour mettre en valeur les compétences linguistiques et philologiques de Brasavola. Ce qu'il faut ici souligner, c'est que la philologie n'est pas une fin en soi pour les médecins de Ferrare. Elle est mise au service d'une pratique médicale ${ }^{39}$. L'établissement du meilleur texte possible prend sens dans le cadre d'un exercice de la médecine et la fiabilité du texte est la condition d'une pratique assurée et certaine.

\section{Expliquer Galien par Galien}

Les savants de l'Occident médiéval connurent essentiellement la médecine galénique par le filtre d'Avicenne et la philosophie d'Aristote par celui d'Averroès. Un des crédos des maîtres de Ferrare, reprenant le vieil adage d'Aristarque ${ }^{40}$, fut d'expliquer Galien par Galien et non par le truchement des commentateurs arabes et médiévaux. Dans son Antisophista ${ }^{41}$, Leoniceno proclame :

Galenus siquidem ex Galeno est intelligendus. Caetera omnia sunt nugae, et falsae latinorum expositorum qui Arabes in plerisque sunt imitati, imaginationes.

[Il faut expliquer Galien par Galien. Tout le reste est sottise et inventions mensongères des commentaires latins qui ont imité à maintes reprises les Arabes.]

Cette perspective de retour aux textes de Galien trouve sans doute son couronnement dans la personne de Brasavola, futur concepteur de l'Index galénique. Dans ses Commentaires et annotations, Brasavola élucide tout d’abord les références croisées que tisse Galien à l'intérieur de ses propres œuvres. En I 29 G (sectio XXX), Galien écrit :

Comment il faut juger si une substance est froide ou chaude, nous l’avons évoqué en ce qui concerne les êtres animés dans le traité Sur les 
tempéraments et, en ce qui concerne les inanimés, dans le traité Sur les facultés des médicaments simples. ${ }^{42}$

Le commentaire de Brasavola apporte quelques précisions à ces références bibliographiques :

Lis le deuxième livre du Sur les tempéraments à peu près à la fin. Une méthode y est donnée pour trouver quel homme est le plus tempéré et ensuite quels sont les tempéraments de chaque partie du corps. Mais il expose aussi les tempéraments dans le troisième livre Sur les médicaments, même si l'intention première de ce livre-là est de parler de l'homme. $\mathrm{Au}$ sujet des êtres inanimés, le livre que je viens de citer en traite, tout comme parmi les livres du De simpl. medic. facul., il est un endroit en particulier où l'on apprend de manière générale comment reconnaître les tempéraments des simples. ${ }^{43}$

Sa lecture minutieuse du CRMA l'invite à de multiples renvois aux autres œuvres du corpus galénique. Le traité le plus fréquemment cité est le De methodo medendi, mais Brasavola mentionne aussi des traités nosologiques (De crisibus, De symptomatum causis), pharmacologiques (De simplicium temperamentis, De remediis parabilibus, De compositione medicamentorum sec. loc.) et surtout diététiques (De alimentorum facultatibus, De sanitate tuenda, De victu attenuante, De ptisana). Pour ce dernier traité, Brasavola émet à juste titre l'hypothèse qu'il soit apocryphe ${ }^{44}$, ce qui montre la facilité avec laquelle il discerne le Galien authentique du pseudo-Galien.

Enfin, il fait preuve d'une parfaite connaissance du texte qu'il commente et sait guider son lecteur à travers les méandres du commentaire galénique. Dans son exégèse à I 43 G (= sectio XLIV, p. 92, col. b, 1. 22-29), il précise :

Sache que Galien, en maints endroits de cet ouvrage a fait mention de ceux qui remplissent trop les malades ou les rétablissent par un régime trop léger. La première mention se situe plus haut à la section 13 et à la section 25. Ajoute, pour le livre II, que dans la section 13 il y a un exposé sur la privation de nourriture, puis ajoute les sections $15,34,40,43,44,47$, 48 et 54 ; pour le livre III, les sections 17,18 et pour le livre IV, la section 5 
et la section 13, qui traite de ceux qui subiront la coction le cinquième ou le septième jour, puis les sections 18 et $44 .^{45}$

Point de doute, l'initiative de commenter le CRMA et sa réalisation par Brasavola font des Commentaires et annotations une des plus belles illustrations du galénisme triomphant des années 1540. Toutefois, l'érudition de Brasavola impressionne également par la somme de ses références aux classiques.

\section{La culture classique de Brasavola}

Le médecin humaniste fait appel à des grands textes littéraires de l'Antiquité. Il cite Horace, Martial, Juvénal ou Vitruve, il se réfère à l'Anabase ou aux Helléniques de Xénophon ${ }^{46}$ dans le but de fournir des realia illustrant les conceptions antiques des textes commentés. Il s'appuie aussi abondamment sur les textes de philosophie naturelle d'Aristote (Histoire des animaux, Sur la génération et la corruption, Sur la génération des animaux, Problèmes) et mentionne les Problemata d'Alexandre d'Aphrodise. Parmi les textes techniques latins, il se réfère aux textes des agronomes (Caton, Palladius), des médecins (Caelius Aurélien, Celse) et surtout à l'encyclopédiste Pline. Les principaux médecins grecs et byzantins sont mentionnés : Dioscoride, Archigène, Oribase, Alexandre de Tralles, Aétius ou Paul d'Égine. En ce sens, Brasavola poursuit la tradition médicale de son maître Manardi qui cherchait à comprendre comment les Grecs lisaient eux-mêmes leur texte et qui affirmait qu'un ancien comme Galien était un bien meilleur exégète que tous les commentateurs modernes ${ }^{47}$.

Outre la masse de références érudites qui est rassemblée, c'est bien aussi par ses méthodes que Brasavola affiche la modernité de son approche philologique. On remarque qu'il utilise à deux reprises les notices biographiques de la Souda au sujet du poète comique Eupolis ${ }^{48}$ et de Dexippe ${ }^{49}$, un des disciples d'Hippocrate. Une des grandes qualités méthodologiques de Brasavola réside dans la constitution, à partir d'un simple mot, d'un véritable dossier qui collecte ses occurrences et ses emplois dans les textes gréco-latins. Ce recensement des occurrences n'a toutefois rien d'un catalogue. Le matériau collecté vise à faire dialoguer les témoignages, à approcher du sens précis d'un mot, à faire émerger les thèses en présence. Les plus longues de ces fiches constituées par Brasavola sont aisément repérables dans le livre, puisqu'elles sont annoncées par un titre 
qui se détache du corps du texte : de garo, de melicrato, de pane, de ouis, de uenae dissectione in pleuritide, de uinorum coloribus etc.

À titre d'exemple, on peut s'intéresser à la fiche sur le garum ${ }^{50}$ (I $17 \mathrm{G}=$ sectio XVIII, p. 41, col. a, 1. 5-p. 43, col. b, 1. 42). Brasavola débute par la mention du garum chez Galien et Dioscoride, mais assez vite, il déplore qu'on ne puisse trouver une véritable recette de cette préparation à base de poissons : puisque tout le monde la connaissait, il n'y avait nul besoin d'en faire état. Brasavola note d'abord que le genre du mot est incertain, tantôt masculin, tantôt neutre, avant de répertorier toutes les sortes connues de garum ${ }^{51}$. Le médecin de Ferrare construit son raisonnement en s'appuyant sur Pline, Martial, Caelius Aurélien, Ausone, Apicius, les Geoponica (attribués à Constantin César) ou Alexandre d'Aphrodise. Il note qu'en général, cette sauce est confectionnée à base d'intestins de scombre ou de maquereau et parvient finalement à trouver deux recettes, l'une dans les Geoponica, l'autre dans le livre III du De remediis facile parabilibus qu'il juge apocryphe. Enfin, il explique que cette enquête lui a permis de corriger son idée première :

Avant, j'étais dans l'erreur, croyant que ce que nous nommons caviar était le garum des anciens, alors que ce n'est pas le cas. De fait, le garum est fait à partir des intestins, alors que le caviar provient des œufs des poissons (...). Mais le caviar est préparé à partir d'œufs de toutes sortes de poisson et les cuisiniers de notre très illustre prince Hercule, duc de Ferrare, préparent plus excellemment le caviar qu'on ne saurait le dire. ${ }^{52}$

La vaste érudition classique et les méthodes éprouvées de Brasavola en matière de philologie ne déçoivent en rien l'idée que l'on pourrait se faire de l'humanisme médical ferrarais. En revanche, la connaissance et l'utilisation des sources arabes est plus surprenante chez un disciple de Leoniceno. Pour le chercheur du XXI ${ }^{e}$ siècle, cet aspect du commentaire de Brasavola est précieux car l'introduction de sources arabes pour interpréter Galien apporte des informations sur la réception du médecin de Pergame dans le monde arabe et sur la synthèse arabo-latine du galénisme tel qu’il s'est déployé en Occident. 


\section{Confronter les Grecs et les Arabes}

L'école de Ferrare est considérée comme le bastion et le foyer de propagation d'un fort courant anti-arabiste ${ }^{53}$ à la Renaissance. Brasavola s'inscrit dans cette veine de dénigrement de la médecine arabe, mais sa démarche n’a rien de dogmatique et sa position par rapport aux auteurs arabes est plus subtile qu'on ne pourrait le penser. Contrairement à ses maîtres, Brasavola ne dévalorise pas systématiquement les médecins arabes. Il hérite bien d'un discours tout fait qui considère les auteurs arabes comme mineurs et barbares, discours dont témoignent par exemple les derniers mots de son introduction :

\footnotetext{
Voici la raison pour laquelle nous avons abandonnéla coutume en usage qui veut que celui qui accède à cette chaire prenne les auspices, en interprétant le quatrième livre du Canon d'Avicenne. Cette coutume, je m'en acquitte à présent autrement : Avicenne fut certes un grand et honnête homme, mais pas autant que ce qu'il faut accorder en comparaison à Hippocrate et Galien. De plus, nous poursuivons ce que nous avons institué en nous mettant à l'école des œuvres d'Hippocrate et naturellement de Galien, ainsi qu'en les interprétant publiquement grâce à tous les autres auteurs de second ordre et en particulier grâce aux Arabes..$^{54}$
}

Si Brasavola établit une hiérarchie entre Galien et Hippocrate d'une part et les auteurs arabes d'autre part, dans ce discours programmatique, on constate qu'une place est indéniablement accordée à ces derniers.

\section{Bannir les barbarismes}

Comme Leoniceno et Manardi, Brasavola s'évertue à assainir, clarifier et stabiliser la terminologie médicale en bannissant les mots décalqués de l'arabe et en condamnant les anciennes traductions médiévales. Quand il cite la vetus translatio de Gérard de Crémone, il entoure cette citation de précautions oratoires :

Et is qui antiquitus hunc librum è graeco in latinum vertit hanc lectionem habere visus est, quippe dicit, licet barbare... 
[Celui qui jadis traduisit ce livre du grec au latin ${ }^{55}$ semble avoir eu cette leçon, puisqu'il dit, bien que la langue soit barbare... ]

Ubi vides in his barbaris verbis...

[Où tu vois à travers ces mots barbares... ]

(I $42 \mathrm{G}=$ sectio XLIII, p. 90, col. b, 1. 14-19).

Dans cette ancienne traduction, le mot ỏ $\xi \dot{\mu} \mu \varepsilon \lambda_{\mathrm{l}}$ était rendu par le terme «secaniabin », tandis que Brasavola use du décalque grec oxymeli.

Dans sa notice sur le garum, Brasavola répertorie une recette tirée du troisième livre du De remediis facile parabilibus qu'il juge étrange car elle n'est pas à base d'intestins de poissons. Dans ce cadre, il émet l'hypothèse intéressante que ce livre III, attribué à Galien ait des sources arabes :

Je ne puis m'abstenir de mentionner une recette de garum attribuée à Galien, dans le troisième livre, attribué également au même auteur, du Sur les médicaments faciles à se procurer, recette dont nous ignorons pourquoi elle porte le nom de garum, puisque ce garum ne comporte nulle trace de poisson. En revanche, la recette utilise d'obscurs noms de mesure : il s'agit plutôt d'une recette arabe que d'une recette composée par un Grec. Elle porte un titre : « composition du garum par Joachim de Martyropolis ${ }^{56}$

Cette recette, parce qu'elle n'est pas le vrai garum des anciens, qu'elle n'est pas de Galien et qu'elle provient du monde arabe, ne mérite pas qu'on s'y attarde :

Assurément, si ce condiment avait été de Galien ou de quelque homme de qualité, je me serais efforcé de toutes mes forces d'interpréter nombre de ses obscurités. Mais étant donné qu'elle n'émane pas d'un auteur digne de foi et qu'elle ne correspond pas au garum des anciens, qui était fait à base d'intestins de poissons et de sel, je ne m'attarderai pas à éclairer ces passages obscurs. ${ }^{57}$ 


\section{Avicenne détrôné?}

Dans son Examen omnium simplicium medicamentorum publié à Lyon en 1537, Brasavola remet en cause le titre de "prince des médecins " que portait naguère Avicenne : "Quid de Avicenna ? Non quidem principem medicorum (ut recentes putarunt) sed Cordubae ${ }^{58}$. Avicenne se voit ainsi privé de sa couronne de premier des médecins, pour n'être plus que le premier de Cordoue.

À la fin de son introduction aux Commentaires et annotations, dans un passage cité plus haut, Brasavola explique aussi qu'il a remplacé la traditionnelle lecture du livre IV du Canon par un commentaire au CRMA de Galien. Le premier fen du livre IV du Canon était l'un des textes universitaires fréquemment étudiés depuis le Moyen Âge. Cette section traite des fièvres et il y a donc une continuité thématique certaine entre le commentaire de Galien et le texte arabe auquel il se substitue, puisque les maladies aiguës sont justement caractérisées par une fièvre intense et continue. Durant le Moyen Âge, ce fen du Canon avait une place capitale dans l'enseignement de la médecine pratique : le cours sur la théorie des fièvres consistait ni plus ni moins en un commentaire de ces pages d'Avicenne. Au XVI ${ }^{\mathrm{e}}$ siècle, cette tendance se poursuit ${ }^{59}$ : le fen $1 \mathrm{du}$ livre IV du Canon fut, par exemple, édité dans l'Articella padouane de $1506^{60}$. Toutefois, pour enseigner les fièvres, on se réfère aussi désormais au De febrium differentiis de Galien et N. Siraisi ${ }^{61}$ constate qu'à Ferrare, les lectures du Canon s'éclipsent devant celles des textes hippocratiques des années 1540 à la fin du $\mathrm{XVI}^{e}$ siècle, mais qu'elles ne disparaissent pas complètement puisquen 1611, le Canon fait à nouveau partie des œuvres lues par le premier professeur ordinaire de theoria médicale.

Ily a manifestement unevolonté et uneidéologiedansles choix deBrasavola, mais la priorité donnée aux textes grecs ne doit toutefois pas occulter sa grande connaissance des médecins et des philosophes arabes. Pendant longtemps, on s'est avant tout intéressé au Canon parce qu'il transmettait les théories galéniques. Tous s'accordaient pour reconnaître dans la somme d'Avicenne une présentation du système galénique plus claire, plus compréhensible et mieux adaptée à la pratique médicale que les textes de Galien eux-mêmes ${ }^{62}$. Le Canon était utilisé comme une synthèse des enseignements galéniques réagencés et simplifiés, d'où l'incroyable fortune de ce texte à la Renaissance, en dépit de la redécouverte du Galien grec. Cette permanence du Canon d'Avicenne dans les programmes universitaires médicaux peut être illustrée par les propos d'un 
contemporain de Brasavola, qui fut lui aussi un disciple de Nicolò Leoniceno : Giovan Battista Da Monte (1489-1551). Cet éditeur des opera omnia du Galien latin, parues en 1541 à Venise chez Lucantonio Giunti et plus connues sous le nom de " juntine ", glisse en effet un éloge chaleureux d'Avicenne dans son épître dédicatoire ${ }^{63}$. Il loue surtout le philosophe et médecin musulman d'avoir rassemblé des données disséminées dans les différents livres de Galien en un tout organisé et cohérent pour en faire un véritable manuel d'enseignement. Selon $\mathrm{Da}$ Monte, le style inélégant du Canon, tant décrié par les médecins hellénistes, s'explique par la mort prématurée d'Avicenne, qui a laissé l'œuvre dans un état inachevé de rédaction ${ }^{64}$.

Brasavola, dans ses Commentaires et annotations, semble également adhérer à ce vieux rêve médiéval de conciliation des Grecs et des Arabes. Dans cette mesure, par les comparaisons systématiques que Brasavola établit entre les médecins grecs et arabes, tout comme par l'application des méthodes critiques de la philologie grecque aux sources arabes, le médecin de Ferrare fait figure de précurseur. En recherchant avec précision les lieux de confrontation entre Avicenne et Galien, Brasavola semble par exemple devancer le travail éditorial d'un Benedetto Rinio, auteur d'une nouvelle édition du Canon en $1555^{65}$, dans laquelle il relevait l'ensemble des passages où Avicenne se référait à Galien.

\section{Le dialogue entre les Grecs et les Arabes}

L'une des ressources insoupçonnées des Commentaires et annotations réside dans le grand nombre d’auteurs arabes qui sont mobilisés. Dans son commentaire au lemme hippocratique sur la ptisane I $17 \mathrm{H}$ (= XVIII), Brasavola restitue une controverse qui avait cours chez les médecins du monde musulman à propos du type d'orge à utiliser pour sa préparation. La ptisane qui est le thème central du livre I du RMA hippocratique, est une décoction d'orge mondé que les anciens considéraient à la fois comme un aliment et comme un remède adapté aux malades souffrant de maladies aiguës. Les médecins arabes polémiquaient pour savoir s'il fallait préparer la ptisane à partir des grains d'orge entiers ou broyés (nonnulli volunt confractum esse opportere hordeum, alii integrum):

Avenzoar in primo theisir tractatu .16. capite .6. qui inquit in unoquoque semine uirtutem esse naturalem antequam frangatur, et in frusta 
conteratur. unde hi à recto aberrant tramite, qui hordeum conterunt... ... eandemque rationem secutus est Auerrois in quinto collectionum libro, capite trigesimoprimo ... Alij Arabes, ut Isaac in diaetis particularibus uolunt hordeum debere esse confractum, et Azarauius libro .26. cap .1. addit terendum esse in mortario lapideo, quamuis Aliabas quinto theoricae dicat moderate terendum esse... At si intentionem illorum consideremus, de ptisana loquuntur, Auicenna et ipse in quarto canone ... iubet hordeum non solum in frusta, sed et in farinam conterendum... (I $17 \mathrm{H}=$ XVIII, p. 26, col. b, 1. 8-26).

Le dossier réuni par Brasavola montre l'étendue de son érudition. Il cite successivement Avenzoar, c'est-à-dire Abu> Marwa>n Ibn Zuhr (1092/5-1161) et son Taysîr fî l-muda>wa>t wa-l tadbîr (Manuel des traitements et régimes), puis Averroès, c'est-à-dire Ibn Rushd (1126-1198) et son Colliget (Kita>b alkulliyya $>\mathrm{t}$, Le livre des généralités), comme partisans du grain d'orge entier. Trois autres médecins arabes défendent à l'inverse l'orge broyé : Isaac Israeli (Ish\}a>q al-Isra>'îlî, 858-955) dans ses Diaetae particulares, Azaravius, c'està-dire al-Zahra $>$ wî ( $\mathrm{X}^{\mathrm{e}}-\mathrm{XI}^{\mathrm{e}}$ siècle) dans son Kita $>\mathrm{b}$ al-Tas\}rîf li-man 'adjiza 'an al-ta'lîf (Livre du classement [de la connaissance médicale] pour celui qui est incapable de compiler [ un manuel pour lui - même]), enfin 'Alî b. al-Abba>s al$\operatorname{Mag} \wedge \mathrm{u}>$ sî et son célèbre Pantegni (Ka>mil as\}-s\}\}ina $>$ 'a). Après avoir tranché ce débat, en expliquant que, pour sa part, il suit Galien, Avenzoar et Averroès, Brasavola cite d'autres témoignages grecs, cette fois-ci, en mentionnant Oribase et les Géoponiques.

Ailleurs, le médecin ferrarais note une contradiction entre Galien et Avicenne à propos du régime des lutteurs ${ }^{66}$ : tandis que Galien préconise dans le $C R M A$ du pain seul le matin et de la viande le soir, Avicenne au $3^{\mathrm{e}} \mathrm{fen}$ du livre I, VII, 2, prescrit un régime carné le matin et du pain seul le soir. Face à ces deux prescriptions diamétralement opposées, Brasavola embrasse le parti de Galien, mais suggère que le texte d'Avicenne est erroné en raison d'une faute d'inversion due aux copistes.

Plus loin, Brasavola expose une autre opposition cette fois entre Galien et Averroès ${ }^{67}$. Brasavola était du reste un fin connaisseur d'Averroès, comme nous le rappelle Ernest Renan, dans son Averroès et l'averrö̈sme : "La bibliothèque de Ferrare ( $\left.n^{\circ} 304\right)$ possède le manuscrit autographe des commentaires inédits 
du médecin Antoine Brasavola sur Averroès, dédié à Hercule d'Este et Renée de France. $»^{68}$

Galien interdisait de donner de l'eau froide aux malades souffrant de fièvre avant que la nourriture ingérée n'ait subi la coction. En effet, l'eau froide pouvait entraver et ralentir le processus de digestion-coction et dès lors, laisser dans le corps des humeurs et des aliments crus, susceptibles de se putréfier et de faire croître la fièvre. Pour Galien, il fallait donc attendre la coction avant de donner de l'eau froide. Mais Averroès n'était pas de cet avis. Au livre VII, c. 8 de son Colliget, il objecte qu'il ne faut pas attendre la coction, car pendant ce temps le malade se consume et que cette consomption entraîne une mauvaise coction. À l'appui de cette thèse, Averroès cite deux exemples qu'il emprunte à Razi :

Averroès ajoute aussi l'exemple, exposé par Razi, de deux jeunes gens, qui faisaient route par des jours caniculaires si bien que chacun des deux attrapa le causus : au maitre de l'eau fut donnée et il fut guéri, mais à l'esclave, on n'en donna pas et il mourut ${ }^{69}$ (I $42 \mathrm{G}=$ XLIII, p. 88, col. b, 1 . 67-p. 89, col. a, 1. 2).

Brasavola explique qu'il s'est longtemps abstenu de donner de l'eau froide, en suivant les préceptes galéniques, jusqu’au jour du 16 février 1545 où il en donna deux fois à un malade avec succès. On voit donc que dans sa pratique, Brasavola a pu au moins une fois suivre Averroès contre Galien ${ }^{70}$. Les grands textes médicaux arabes dialoguent donc avec les sources antiques : ces deux types de témoignage sont mis sur le même plan au sein d'un débat et Brasavola peut parfois donner sa préférence aux Arabes. En tout cas, il faut saluer cet effort de méthode chez Brasavola : il met ses sources à distance, et les utilise avec un œil critique. La culture de Brasavola n’a donc rien de monolithique. Médecin helléniste, il manifeste un intérêt pour la science arabe ${ }^{71}$. Dans une certaine mesure, il participe au renouveau des études arabes qui se développe au XVI ${ }^{e}$ siècle et qui prendra son plein essor au XVII ${ }^{\mathrm{e} 2}$. 


\section{III. Être homme de son temps}

Cette érudition poussée ne doit pas donner l'image d'un médecin antiquaire, uniquement tourné vers le passé et fermé à la nouveauté. Les références bibliographiques de Brasavola montrent qu'il connaît aussi les travaux de ses contemporains : Érasme, Giorgio Merula, Ermolao Barbaro ou encore Guillaume Cop. De plus, l'admirable savoir livresque qui est exhibé dans le commentaire se combine avec une réelle expérience pratique de la médecine. En suivant et en soignant des patients, Brasavola est en mesure de juger, voire de contester les données des anciens.

\section{Travaux d'érudits}

Brasavola est bien au fait des travaux philologiques menés sur les classiques aux $\mathrm{XV}^{\mathrm{e}}$ et $\mathrm{XVI}^{\mathrm{e}}$ siècles. En I 17 G (= sectio XVIII, p. 42, col. a, 1. 22 sq.), il cite l'interprétation que fit Giorgio $\mathrm{Merula}^{73}$ en rencontrant l'expression garum sociorum chez le poète latin Ausone, quoique pour la réfuter. Un peu plus loin, à propos d'un passage corrompu chez ce même Ausone, Brasavola mentionne que le locus deprauatus a été signalé par Hermolao, c'est-à-dire par l'humaniste Ermolao Barbaro ${ }^{74}$ (I $17 \mathrm{G}=$ sectio XVIII, p. 43, col. b, 1. 5-7). Mais c'est principalement avec son contemporain Guillaume Copp ${ }^{75}$, médecin de François I ${ }^{\text {er }}$, ami d'Érasme, de Budé et de Lefèvre d'Étaples et traducteur de Paul d'Égine, que Brasavola instaure un dialogue dans ses Commentaires et annotations. En effet, Copp avait publié dès 1511 à Paris, une traduction du Régime des maladies aiguës hippocratique ${ }^{76}$. Brasavola utilise cette traduction quand il commente les lemmes, le plus souvent pour signaler son désaccord :

Le sens que donne ici Copp n'est pas « que l'on augmente la nourriture après deux jours », mais « que l'assaut de la crise n'excède pas, en général, deux jours ». Mais nous disons qu'il ne doit pas même excéder un jour. ${ }^{77}$ 


\section{L'expérience d’un praticien}

Jusqu'à présent, l'étude des Commentaires et annotations esquisse le portrait d'un médecin lettré, féru de livres, de textes et de mots, mais ce ne serait pas rendre justice à l'auteur que de s'en tenir là. Le commentaire prend corps et sens à travers la multitude de notations personnelles qui le nourrissent. Ce qui frappe dans ces observations de Brasavola, c'est leur précision. À propos des poissons de roche, que Galien recommandait vivement aux malades comme un aliment léger et salutaire, Brasavola fait cette remarque, tant zoologique que géographique, qui témoigne d'un sens aigu de l'observation de la nature :

Dans les villes proches de Ferrare qui se situent près des montagnes, on trouve une variété d'espèces de poissons véritablement saxatiles d'après le lieu où ils habitent, comme à Bologne, Florence, Sienne, Lucques, Modène ou Reggio d'Émilie. ${ }^{78}$

$\mathrm{Au}$ début des Commentaires et annotations, il explique qu'il a un jour entendu quelque médecin défendre de toutes ses forces que nul n’avait écrit sur la médecine avant Hippocrate et que lui-même avait entrepris de réfuter ce médecin en citant le premier lemme hippocratique où sont mentionnées les Sentences cnidiennes. Brasavola donne des précisions sur cette scène : elle se déroulait à Lucques, au mois de septembre 1541, à l'heure où le Pape déjeunait ${ }^{79}$. Le commentaire est parsemé de semblables notations ${ }^{80}$ qui révèlent un esprit curieux, mais surtout une volonté d'enregistrer précisément un témoignage référencé dans l'espace et dans le temps, comme pour être utile aux générations à venir.

En tant que médecin, Brasavola consigne également son expérience personnelle. Quand Galien et Hippocrate évoquent les maladies pestilentielles, Brasavola se souvient de la grande épidémie de peste qui ravagea toute l'Italie en 1528 et il fait mention des formes successives que prit la maladie sur le corps des pestiférés (I $8 \mathrm{G}=$ sectio IX, p. 13, col. b, 1. 59-p. 14, col. a, 1. 4). Peu après ce développement, il donne un exemple de maladies endémiques à travers la formation du goitre qui serait dû à un environnement trop humide. Ici encore, Brasavola étonne par l'exactitude de sa description : 
Dans les Alpes, qui séparent l'Italie de la France, en un lieu nommé Moriana, ainsi appelé en raison d'un fleuve boueux, nous vîmes un prêtre qui avait un goitre d'un poids de vingt livres, à ce qu'estimaient tous ceux qui étaient en compagnie du très illustre Hercule, duc de Ferrare, mais moi, au vu de sa dureté et de sa densité, je l'estimais à vingt-cinq livres. ${ }^{81}$

La pratique et l'expérience médicale de Brasavola transparaissent tout au long du commentaire. J'ai déjà mentionné que la rédaction des Commentaires et annotations pouvait être mise en relation avec l'obtention par Brasavola de la chaire de médecine pratique en 1541, puisque leur rédaction débute en 1542. Dans le prologue, il inaugure son commentaire en relatant, un peu à la manière de Galien, une anecdote pleine d'ironie à ce sujet :

Nous avons entendu certaines personnes sur la place publique ou chez les parfumeurs grommeler ou à l'inverse s'ébahir d'admiration de ce que nous ayons l'audace, à un âge si immature, à ce qui leur semblait, d'étudier dans les écoles publiques cette partie de la faculté de médecine qu'on nomme praxis : l'apanage d'expliquer cette partie de la médecine devrait revenir aux gens plus âgés, déjà accablés sous le poids de l'âge et consumés par la vieillesse. ${ }^{82}$

Il faut ici signaler l'existence, dans un manuscrit de la Bibliothèque municipale de Ferrare, d'un recueil de traitements de malades, qui se présente par fiches, comme les Épidémies hippocratiques : Curationes A. M. Brasauoli. ${ }^{83}$ Dans ce recueil, Brasavola soigne 84 patients différents atteints de pathologies variées. Le document date vraisemblablement des années 1547-1548. Il semble qu'il s'agisse de notes d'élèves qui suivaient Brasavola dans ses consultations : les fiches sont rangées dans un ordre chronologique et elles permettent de suivre le travail du médecin qui se rendait deux fois par jour au chevet des malades, parfois même la nuit. Chaque thérapie est soigneusement retranscrite. On peut donc mettre en relation le choix du CRMA pour son cours de médecine pratique et cet exercice quotidien de la médecine auprès des malades, même si le document de Ferrare est un peu postérieur à la rédaction et à la publication du commentaire. Quoi qu'il en soit, c'est en vertu de cette pratique médicale empirique que Brasavola questionne la diététique hippocratico-galénique. 


\section{La fin du régime hippocratique}

De toute évidence, Brasavola accepte la diététique comme une des branches de la thérapeutique. Elle a sa place au même titre que la chirurgie et la pharmacologie ${ }^{84}$. Étant donné que le traité commenté porte sur le régime, l'exégète fournit un grand nombre d'informations sur les usages alimentaires de son époque. Quand il est question chez Galien de soupe de lentilles, Brasavola explique qu'on l'appelle communément à Ferrare favetta ou favetta da frati ${ }^{85}$; à propos du pain, il fait allusion à l'usage ferrarais de cuire sous une tuile des tortae ou des focacei. Si le commentateur tente d'établir des correspondances entre le passé et le présent, il souligne aussi parfois des différences. Il explique qu’à son époque, le sucre a remplacé le miel :

Nous utilisons de nos jours un condiment plus délicat. Nous avons en effet le sucre, inconnu des anciens, avec lequel nous préparons l'oxyzaccar pour cet usage. Le sucre est presque tempéré et il ne cause pas de flatuosité comme le miel. ${ }^{86}$

Le principal problème concerne la ptisane. C'est autour de cette décoction d’orge mondé que s'organise le livre I du CMRA : Galien et Hippocrate en ont fait l'aliment idéal pour les patients atteints de maladies aiguës. Pour Hippocrate, la ptisane a d'abord pour le malade un caractère agréable et plaisant : on l'avale facilement, elle ne sollicite pas la mastication, elle est coulante. Elle tient de lorge son caractère froid et humide, ce qui permet de tempérer la chaleur et la sécheresse des fièvres ${ }^{87}$. Bouillie, elle doit à son mode de préparation sa digestibilité et son innocuité dans le processus de $\operatorname{coction}^{88}$. Pour Galien, la ptisane possède en prime la vertu de diviser et de fluidifier les amas d'humeurs et de matière qui entravent le bon fonctionnement de la digestion et la circulation des fluides dans le corps. Pour les deux médecins, la ptisane est donc à la fois un aliment et un médicament. En tant qualiment elle a le mérite d’apporter de la force au malade, sans trop augmenter la chaleur innée du corps, déjà accrue du fait de la fièvre. Pour les médecins anciens, le processus de digestion était perçu comme une cuisson, lors de laquelle les aliments passaient de l'état cru à l'état cuit. Dans ce mécanisme, la nourriture était considérée comme une sorte de combustible. Quand, lors des fièvres, la chaleur innée était déjà très élevée, il fallait absolument éviter d’augmenter la température du corps en ingérant 
des aliments trop lourds ou trop indigestes : d’où les vertus de la légèreté de la ptisane qui pouvait maintenir les forces vitales du patient, sans pour autant faire monter la température du corps. La ptisane était utilisée à l'époque d'Hippocrate comme un aliment quotidien, mais ce n'était déjà plus le cas au temps de Galien ni a fortiori à la Renaissance. Dès l'époque romaine, on préféra le blé à lorge et le pain aux bouillies. Si donc la préparation à base d’orge mondé est restée en honneur de l'Antiquité à la Renaissance ${ }^{89}$, cest uniquement grâce à l'autorité d'Hippocrate et de Galien. Brasavola donne toutefois quelques informations sur ce que ses contemporains pensaient de la ptisane :

Force est de s'étonner que certains de nos contemporains prétendent qu'il n'existe pas de bonne orge, parce que, pour nous, il s'agit d'une nourriture pour les bêtes. Selon eux, il n'est pas possible à notre époque de faire de la ptisane parce qu'il n'existe pas de cuisiniers qui sachent la préparer; enfin, puisque c'est une nourriture pour les bêtes, nous ne manifestons, à dire vrai, aucun soin en semant, en récoltant et en conservant l'orge. ${ }^{90}$

À ces allégations, Brasavola répond qu'il existe de l'orge de bonne qualité à son époque et qu'il est encore possible, sans aucun doute, de préparer une excellente ptisane. Plus loin, il répond à cette même critique à l'égard de l'inadéquation de l'orge et de la ptisane :

Parce que nos femmes, à la maison, comme nous le leur avons appris par le passé, préparent remarquablement la ptisane et qu'elle est délicieuse et agréable, nous prenons très fréquemment, au lieu du blé commun, de la ptisane en guise de plat léger et toute notre famille, ainsi que nos hôtes, en mangent souvent, en la savourant. Pendant les jours où la viande est proscrite, les femmes de la maison la préparent avec de l'huile, du sel, du poireau, de l'aneth et un peu de vinaigre - à mon goût, elle semble toutefois plus savoureuse sans vinaigre. Il faut dès lors penser qu'ils n'ont pas eu de chance les médecins qui n'ont pas su enseigner à leurs cuisiniers comment il fallait la préparer. Car après l'avoir enseigné une seule fois à une faible femme, nous l'avons instruite de sorte que jamais elle ne la rate. ${ }^{91}$ 
L’expérience personnelle de Brasavola de ptisanes réussies n’implique pourtant pas que le médecin reprenne les théories médicales antiques sur la décoction. Loin s'en faut. Il explique en effet que le goût des patients a changé et qu'il n'est pas question de leur imposer un aliment qu'ils n’apprécient plus :

Pourtant, à notre époque, les médecins ne peuvent suivre cette progression dans les maladies aiguës, parce que rares sont ceux à qui plaisent le jus de ptisane ou la ptisane, mais nous sommes forcés, bon gré mal gré, de prescrire de l'eau d'orge (...). Pourtant ceux auxquels plaît la ptisane, nous leur donnons de la ptisane et du jus de ptisane. Néanmoins les produits que nous proposons ne doivent pas déplaire. ${ }^{92}$

Le traitement par la ptisane n'est donc pas toujours possible. Brasavola va même jusquà critiquer la prescription de jus de ptisane dans certains cas :

Une fois établi que la crise est passée, il faudrait sans aucun doute prescrire une nourriture plus copieuse et d'un autre genre que la ptisane ; en réalité, à la place de la ptisane, nous donnons du " pain lavé », concocté dans un extrait de graines de pastèque ou d'autres choses du même genre, puisque les Ferrarais ne sont pas habitués à la ptisane, même si, bien préparée, elle est savoureuse au goût. J'ai plus en horreur le jus de ptisane que la ptisane entière. $^{93}$

Dans ce passage, plusieurs points sont importants. On retrouve l'idée que les Ferrarais ne sont pas habitués à la ptisane. Mais deux remarques semblent plus surprenantes. D'une part, il faut, après la crise, nous dit Brasavola, un aliment plus copieux et d'un autre genre que la ptisane. Or Galien et Hippocrate établissaient une transition progressive entre le régime de diète sévère avant la crise et l'après-crise : il fallait éviter tout changement brusque. D'autre part, l'exégète manifeste son aversion pour le jus de la ptisane. Ces deux remarques, ajoutées à l'évolution des mœurs alimentaires qui rend difficile la prescription d'un tel régime, n’ont l'air de rien ${ }^{94}$. Mais en réalité, elles remettent en cause les fondements mêmes du traité hippocratique et du commentaire de Galien. Elles sapent l'autorité de ces deux figures en matière de régime thérapeutique. 
On peut rapprocher cette critique diffuse du régime hippocratique pour les malades d'une autre attaque plus virulente. Le médecin portugais Manuel Brudo a ainsi publié en 1544 à Venise, un livre dédié à ses protecteurs anglais Sur le régime des fièvres selon Hippocrate ${ }^{95}$. Arrivé en Angleterre en 1540, ce médecin portugais reprend le régime de Pétronas que Galien avait tant vilipendé $^{96}$ : il est d'avis qu'il faut donner aux patients anglais de la viande rouge et de la bière, parce que ce régime est plus adapté aux contrées froides que la ptisane. Pour Galien, le régime de Pétronas (vin et viande rouges) était l'expression d'une démesure et d'une extrême démence, car il s'opposait en tous points à celui d'Hippocrate, quant à lui mesuré et donc excellent ${ }^{97}$. Les menues critiques émises par Brasavola à l'encontre de la ptisane ne sont pas anodines : elles bouleversent l'équilibre d'un système. Du régime par la ptisane dépend toute une physiologie de la digestion envisagée comme coction ou putréfaction. La ptisane est approuvée par Hippocrate comme un produit quotidien dont l'expérience montre qu'il peut être utilisé avec profit pour nourrir les malades. Cette décoction est par la suite encensée par Galien, sans doute au départ par révérence pour son illustre prédécesseur. Mais Galien fait accéder la ptisane au rang de substance irremplaçable pour réguler les dysfonctionnements physiologiques de la coction et de la fièvre, qui sont étroitement liés. Refuser la ptisane pour des raisons gustatives ou médicales, c'est donc remettre en cause une diététique, et plus largement un système physiologique fondé sur une théorie des fièvres et de la chaleur innée.

Pour clore cette analyse du premier livre des Commentaires et annotations de Brasavola, deux points me semblent devoir être soulignés. Le premier concerne les fiches savantes composées par Brasavola autour d'un mot ou d'une notion. Par le nombre et la diversité des sources rassemblées, ces notices représentent un chef d'œuvre d'érudition galénique. Dans ses enquêtes, Brasavola intègre et mêle les données des traditions grecque et latine, byzantine et arabe, médiévale et renaissante. Rédigées à une époque où n'existaient pas les outils numériques, ces dossiers philologiques sont d'une grande pertinence pour les chercheurs d'aujourd'hui, parce qu'ils concentrent des données qui apparaissent bien souvent atomisées dans la recherche actuelle, mais aussi parce qu'ils demeurent des modèles de méthode.

Une seconde mise en perspective consiste à replacer le questionnement de Brasavola sur la diététique des malades dans une évolution épistémologique. 
Les historiens de la médecine ${ }^{98}$ établissent généralement que le régime hippocratico-galénique perdure jusqu'à l'âge baroque, pour céder ensuite le pas à une nouvelle discipline médicale, du reste mineure, la nutrition. Dans leurs thérapies, les anciens accordaient une part importante à la diététique. Certaines théories antiques considéraient même que l'alimentation était la cause principale des maladies ${ }^{99}$. Humeurs et aliments crus devaient subir la coction car s'ils restaient à l'état cru dans les conduits et les cavités du corps humain, ils créaient des amas, des obstructions, et partant, des maladies. Cette diététique ancienne proscrivait les fruits et légumes ainsi que l'eau, comme néfastes au processus de fermentation-coction. C'est avec les découvertes des iatrochimistes comme Paracelse et son disciple Van Helmont, que le régime grec comme voie thérapeutique est fortement critiqué au profit d'une polypharmacie. Dès lors, la diététique n'est plus une voie de la médecine et les prescriptions alimentaires des anciens ne font plus loi. Au XVIII ${ }^{e}$ siècle, le médecin de Lausanne, S. Tissot ${ }^{100}$, affranchi du poids de la tradition diététique, pourra enfin prescrire pour les fièvres de la soupe de légumes, beaucoup d'eau et des jus de fruits.

Le régime des anciens Grecs s'appuyait sur une physiologie de la digestion et il était lié aux théories des fièvres et de la chaleur innée. Labandon du régime de ptisane n'est possible qu’à partir du moment où le lien entre cette décoction d'orge et les représentations physiologiques se fait moins évident. Dès avant Paracelse, le célèbre érudit allemand Johannes Reuchlin (1455-1522) met en doute l'idée galénique de chaleur innée ${ }^{101}$ : si la chaleur était responsable de la digestion gastrique, la chaleur extérieure pourrait favoriser et améliorer ce processus ; or il n'en est rien. Pour Van Helmont (1579-1644) ${ }^{102}$, la chaleur innée est également secondaire. En étudiant les étapes de la digestion, ce dernier a pu identifier l'existence du suc gastrique. Un des apports de Lavoisier (1743-1794) sera de faire la synthèse des travaux des iatrochimistes et des iatromécanistes pour montrer que la chaleur innée n'est pas un principe vital, mais que la chaleur est communiquée au corps par le sang, comme le résultat d'une combustion chimique qui s'effectue dans les poumons. Progressivement, le système diététique et physiologique des anciens a perdu de son influence. À la fin de l'époque baroque, la médecine n'est plus diététique.

Les textes antiques sur le régime ont cependant trouvé d'autres prolongements que médicaux. Les écrits sur le régime connaissent une postérité par le biais d'un nouveau genre littéraire qui fleurit dès l'époque humaniste et connaît un grand succès jusqu'au XVIII e siècle : il s'agit du régime de l'homo 
literatus ${ }^{103}$. V. Nutton ${ }^{104}$ a, en outre, montré que le De arte gymnastica, publié en 1569 à Venise par Girolamo Mercuriale, avait reçu un accueil tiède de la part des médecins, mais enthousiaste de la part des antiquaires. Ce livre, qui fut assorti d'illustrations dès sa deuxième édition en 1573 et qui tentait d'intégrer à la médecine la pratique d'exercices physiques inspirés de l'Antiquité, a davantage retenu l'attention des amateurs d'imagerie antique et des collectionneurs de médailles que celle du corps médical. Ce n'est que bien plus tard, avec des travaux comme ceux de Jean Trémolières ${ }^{105}$ (1913-1976), que la médecine revient sur l'importance des pathologies dues à la nutrition dans nos sociétés de consommation et que la diététique réintègre le champ des disciplines médicales.

\section{Notes}

1. Sur la complexité de cette assimilation de la médecine grecque à la Renaissance, voir notamment l'étude de Vivian Nutton, «Greek Science in the SixteenthCentury Renaissance ", dans éd. J. V. Field et F. A. J. L. James, Renaissance and Revolution. Humanists, Scholars, Craftsmen and Natural Philosophers in Early Modern Europe (Cambridge: Cambridge University Press, 1993), p. 16-28.

2. Ermolao Barbaro achève sa traduction du De materia medica de Dioscoride en 1481-1482 ; Giorgio Valla traduit le De sectis de Galien en 1483-1484 ; Andreas Brenta publie en 1489-1490 la traduction de plusieurs traités de la Collection hippocratique.

3. Sur la critique de la médecine arabe par les hellénistes, voir P. E. Pormann, « La querelle des médecins arabistes et hellénistes et l'héritage oublié ", dans éd. V. Boudon-Millot et G. Cobolet, Lire les médecins grecs à la Renaissance (Paris : De Boccard, 2004), p. 113-141.

4. Le slogan du médecin néerlandais Jan Van de Velde (1486-1558), « Sans grec, toute médecine n'est qu'imposture » (De pariente et partu, Anwers, 1527, sig. A, f. 5r-v), résume assez bien la tendance qui se dessine durant le second quart du XVI e siècle. V. Nutton estime que cette période durant laquelle pratique de la médecine et philologie grecque ne font qu'une s'achève avec la parution en 1557 à Paris des œuvres du médecin byzantin Jean Zacharias Aktouarios. Après cette date, les éditions de textes médicaux grecs se font rares; voir V. Nutton, « Greek Science in the Sixteenth-Century Renaissance », p. 17. 
5. Dans la seconde moitié du XVI ${ }^{e}$ siècle et au XVII ${ }^{e}$ siècle, Galien perd de son influence au profit d'Hippocrate. Sur ce regain de l'hippocratisme, voir V. Nutton, "Hippocrates in the Renaissance ", dans éd. G. Baader et R. Winau, Die hippokratische Epidemien. Theorie-Praxis-Tradition (Sudhoffs Archiv, Beihefte 27, Stuttgart : Franz Steiner, 1989), p. 420-439 et I. M. Lonie, « The 'Paris Hippocratics' : Teaching and Research in Paris in the Second Half of the Sixteenth Century », dans éd. A. Wear, R. K. French et I. M. Lonie, The Medical Renaissance of the Sixteenth Century (Cambridge : Cambridge University Press, 1985), p. 155-174.

6. Voir V. Nutton, "Greek Science in the Sixteenth-Century Renaissance », p. 16 et du même auteur, «Hellenism Postponed : Some Aspects of Renaissance Medicine, 1490-1530 », Sudhoffs Archiv 81 (1997), p. 158-170, notamment p. 163.

7. Sur le nouveau latin médical à la Renaissance, voir V. Nutton, «The Changing Language of Medicine, 1450-1550", dans éd. O. Weijers, Vocabulary of Teaching Between Middle Ages and Renaissance, Proceedings of the Colloquium London Warburg Institute (11-12 march 1994) (Turnhout : Brepols, 1995), p. 184-198.

8. Sur Antonio Musa Brasavola, voir P. R. Horne, «Reformation and CounterReformation at Ferrara : Antonio Musa Brasavola and Giambattista Cinthio Giraldi », Italian Studies 13 (1958), p. 62-82 ; G. Gliozzi, s. v. " Antonio Musa Brasavola ", Dizionario biografico degli Italiani (Rome : Istituto dell'Enciclopedia italiana, 1972), tome 14: p. 51-52; V. Nutton, « Medicine, Diplomacy and Finance : the Prefaces to a Hippocratic Commentary of 1541 ", dans éd. J. Henry et S. Hutton, New Perspectives on Renaissance Thought. Essays in the History of Science, Education and Philosophy in Memory of Charles B. Schmitt (Londres : Duckworth \& Co-Istituto Italiano per gli Studi Filosofici, 1990, p. 230-243 ; V. Nutton, « The Rise of Medical Humanism : Ferrara, 1464-1555 », Renaissance Studies 11.1 (1997), p. 2-19, spécialement p. 11-17.

9. Des fragments de cette biographie perdue sont conservés dans un manuscrit de la Biblioteca Bertoliana de Vicenza dont la cote est : Gonzaga 543 ; voir V. Nutton, "The Rise of Medical Humanism », n. 3, p. 3.

10. Âgé de 28 ans, il accompagna Hercule II d'Este en France, où celui-ci se rendit pour épouser Renée de France, fille cadette de Louis XII. À Paris, Brasavola rencontra un franc succès. Il soutint pendant trois jours une thèse devant le collège de la Sorbonne qui lui fit l'honneur de l'accueillir parmi ses membres et il soigna le roi François Ir $^{\text {er }}$ qui le décora de l'ordre des chevaliers de Saint-Michel et lui décerna le surnom de Musa, en souvenir du célèbre médecin d’Auguste, Antonius Musa. Dans l'épître dédicatoire de ses Commentaires et annotations, il ranime le 
souvenir de ce voyage en France : Quam uero amplae sint Galliae urbes, quam spatiosae, quam publicis foris, amplis plateis, latis uicis, aedibus ac palatiis mira structura editis decorentur, omnibus palam est, et nos his oculis experti sumus, cum .1528. Illustriss. Herculem Ferrariae Ducem comitaremur. (Qu'elles sont vastes les villes de France! Qu'elles sont spacieuses ! Comme elles sont ornées de places publiques, de vastes avenues, de larges rues, de demeures et de palais édifiés selon une architecture admirable ! Cela s'offrant aux regards de tous, nous avons pu de nos yeux le contempler, lorsqu' en 1528 nous accompagnions le très illustre Hercule, duc de Ferrare). Toutes les traductions des Commentaires et annotations qui suivent sont de mon fait.

11. De utilitate ac necessitate dialecticae, Ferrare, 1527 ; In Porphyrii Isagogas vel quinque voces commentatio, Ferrare, 1530 ; De hominum aequalitate, Venise, 1537 ; Quod nemini mors placeat, Lyon, 1543 ; De ordine et modo iuris discendi, Venise, 1554. Pour élargir l'éventail des centres d'intérêt de Brasavola, on peut aussi ajouter des Commentaria in Pentateucum Moysis inédits qui se trouvent dans un manuscrit conservé par ses héritiers à Ferrare.

12. A. M. Brasavola, Index refertissimus in omnes Galeni libros, Venise, 1551 (pour la première édition).

13. Cet Index latin fut repris partiellement dans le dernier tome de l'édition de Kühn : Claudii Galeni, Opera omnia (Leipzig : Knobloch, 1833), t. XX.

14. Une tradition veut qu'il ait créé en 1536 sur une petite île du Pô, l’un des premiers jardins botaniques ; voir la notice biographique de Gliozzi, "Antonio Musa Brasavola », Dizionario biografico degli Italiani. On sait seulement qu'il rassembla à Ferrare une importante collection de simples et qu'il en recensa plus de 2000. Alessandro Visconti donne un sens à ce chiffre en rappelant qu'Aristote recensait 180 plantes, Théophraste 850, Dioscoride 600 et Pline 1000 ; voir A. Visconti, La Storia dell'Università di Ferrara (1391-1950) (Bologne : Zanichelli, 1950), p. 50-52.

15. Le commentaire de Brasavola est divisé en quatre livres comme, du reste, le commentaire de Galien tel qu'il nous est parvenu dans la tradition manuscrite. Pour une édition critique et une traduction annotée du premier livre du CRMA de Galien, voir A. Pietrobelli, Histoire du texte, édition critique et traduction annotée du livre I du commentaire de Galien au Régime des maladies aiguës d'Hippocrate, thèse de doctorat Paris IV, soutenue en 2008, sous la direction de V. BoudonMillot.

16. Certaines éditrices de Galien ont toutefois manifesté une attention particulière aux commentaires de la Renaissance sur les œuvres du corpus galénique; voir S. 
Fortuna, "Galen's De constitutione artis medicae in the Renaissance ", Classical Quarterly 43 (1993), p. 302-319, ici p. 313-317 ; et plus récemment, B. Gundert, Galeni De symptomatum differentiis, dans Corpus Mecicorum Graecorum (=CMG) V 5, 1 (Berlin : Akademie Verlag, 2009), p. 186-192.

17. Sur l'anti-arabisme de Leoniceno, voir par exemple P. E. Pormann, « La querelle des médecins arabistes et hellénistes et l'héritage oublié », p. 120-122.

18. Giovanni Manardi, Epistolarum medicinalium liber, Bâle, 1540, I, 1, p. 1-2: Quis Auicennam ceu caeleste numen non adorauit? in cuius tamen libro praeter densam caliginem, infinitum esse ambagum chaos, et commentaria quaedam nuper (ut audio) de Auicennae erroribus edita, et alia non multo post edenda, luce clarius (bonis tamen oculis) demonstrabunt. (Qui n’a pas adoré Avicenne comme une déité céleste ? Cependant, des commentaires récemment édités, et d'autres qui doivent encore être édités au sujet de ses erreurs montreront plus clairement que le jour (à ceux qui possèdent de bons yeux) qu'il n'y a dans son livre, outre une obscurité épaisse, qu’un chaos sans fin d’ambiguités). Ce texte est cité et traduit par P. E. Pormann, «La querelle des médecins arabistes et hellénistes et l'héritage oublié », p. 123.

19. Sur cette tripartition de la partie thérapeutique de la médecine, voir Galien, De partibus artis medicae (éd. H. Schöne, dans Festschrift der Universität Greifswald [Greifswald : J. Abel, 1911], p. 23 et M. C. Lyons, CMG, Supplementum Orientale II [Berlin : Akademie Verlag, 1969], p. 25); Celse, De medicina, intr. 9 ; Scribonius Largus, Compositiones, intr. 6 ; Plutarque, De sollertia animalium, 974 b-d ; Diogène Laerce, Vitae philosophorum, III, 85.

20. Les découvertes anatomiques que suscitèrent les dissections de cadavres humains, tout comme l'existence d'une corporation autonome de chirurgiens-barbiers œuvrèrent à émanciper la chirurgie de ses anciens dogmes; voir, entre autres, V. Nutton, "'Prisci dissectionum professores' : Greek Texts and Renaissance Anatomists ", dans éd. A. C. Dionisotti, A. Grafton et J. Kraye, The Uses of Greek and Latin. Historical Essays (Londres : The Warburg Institute, University of London, 1988), p. 111-126 et R. Mandressi, Le Regard de l'anatomiste (Paris : Seuil, 2003).

21. Voir K. M. Reeds, "Renaissance Humanism and Botany ", Annals of Science 33 (1976), p. 519-542 ; R. Palmer, " Pharmacy in the Republic of Venice in the Sixteenth Century ", dans éd. A. Wear, R. K. French et I. M. Lonie, The Medical Renaissance of the Sixteenth Century, p. 100-117. 
22. Sur Georg Agricola, voir entre autres R. Hofmann, Georg Agricola. Ein Gelehrtenleben aus dem Zeitalter der Reformation (Gotha : Perthes, 1905) ; E. Darmstaedter, Georg Agricola 1494-1555, Leben und Werk (Munich : Verlag der Münchener Drucke, 1926) ; H. Hartmann, Georg Agricola 1494-1555 (Begründer dreier Wissenschaften : Mineralogie - Geologie - Bergbaukunde) (Stuttgart : Wissenschaftliche Verlagsgesellschaft M. B. H., 1953) ; H. Prescher, Georgius Agricola. Persönlichkeit und Wirken für den Bergbau und dans Hüttenwesen des 16. Jahrhunderts (Kommentarband zum Faksimiledruck " Vom Bergkwerck XII Bücher” Basel 1557) (Leipzig : V. E. B. Deutscher Verlag für Grundstoffindustrie, 1985).

23. Voir p. 2, col. a, 1. 28-34.

24. Pour le XIII e siècle, sont conservés les commentaires de Taddeo Alderotti, Bernard de Gordon, Petrus Hispanus, Jean de Saint-Amand, Marsilius de Sancta Sophia ainsi que plusieurs autres anonymes.

25. Sur l'Ars commentata, voir C. O'Boyle, The Art of Medicine. Medical Teaching at the University of Paris 1250-1400 (Leyde, Boston, Cologne : Brill, 1998).

26. Sur l'Articella, voir T. Pesenti, «Articella dagli incunabuli ai manoscritti : origini e vicende di un titolo", dans éd. M. Cochetti, Mercurius in trivio, Studi di Bibliografia e di Biblioteconomia per Alfredo Serrai nel 60 compleano (20 novembre 1992) Rome : Bulzoni, 1993), p. 129-145 et J. Arrizabalaga, The Articella in the Early Press c. 1476-1534, «Articella Studies » 2 (Cambridge : Cambridge Wellcome Unit for the History of Medicine, 1998).

27. En évoquant les commentaires de Brasavola, V. Nutton écrit : «He also wrote three huge Hippocratic commentaries that show neatly how the tradition of Leoniceno and Manardi was carried on in the classroom ", dans V. Nutton, "The Rise of Medical Humanism », p. 13.

28. In octos libros Aphorismorum Hippocratis commentaria et annotationes, Bâle, 1541. Sur les préfaces à ce texte, voir V. Nutton, « Medicine, Diplomacy and Finance ».

29. Ce commentaire aujourd'hui perdu est mentionné dans la liste des ouvrages de Brasavola dressée par Jacobus Antonius Bonus dans sa préface à l'Index de l'édition juntine : Scripsit etiam commentaria in Prognostica.

30. Chaque tome a sa propre pagination : le premier comporte 103 pages, le deuxième 106, le troisième 123 et le dernier 253. J'en arrive à un total de 592 pages en comptabilisant les folios blancs non paginés entre les tomes et le folio initial qui porte l'épître dédicatoire, sans les gardes, ni la page de titre. 
31. Sur le commentaire médical comme pratique scolaire, voir D. Manetti-A. Roselli, "Galeno commentatore di Ippocrate », Aufstieg und Niedergang der Römischen Welt II 37, 2 (Berlin, New York : De Gruyter, 1994), p. 1529-1635 et 2071-2080 ; J. Mansfeld, Prolegomena: Questions to Be Settled Before the Study of an Author or a Text, Philosophia Antiqua 61 (Leyde, New York, Cologne : Brill, 1994) et H. Von Staden, "'A Woman Does Not Become Ambidextrous' : Galen and the Culture of Scientific Commentary ", dans éd. R. K. Gibson et C. Shuttleworth Kraus, The Classical Commentary, Histories, Practices, Theory (Leyde, Boston, Cologne : Brill, 2002), p. 109-139.

32. Brasavola, avant d'entreprendre son commentaire, fait allusion à la chaire de médecine pratique qu'il occupe à Ferrare : nos (...) ausos esse, partem illam rei medicae facultatis in publicis scholis aggredi, quae praxis nuncupatur, p. 1, col. a, 1. 4-9 (nous avons l'audace d'étudier dans les écoles publiques cette partie de la faculté de médecine qu'on nomme praxis).

33. Se présentent sous la forme d'un dialogue de Brasavola avec un vieillard l'Examen syrupum (Lyon, 1540) et l'Examen omnium Loch (Venise, 1553). Le traité moral intitulé Quod nemini mors placeat (Lyon, 1543) adopte aussi la forme dialoguée.

34. D. Mugnai Carrara a insisté sur le nouveau type de relations maitre-disciples qu'avait instauré Giovanni Manardi : il refusait un rapport trop hiérarchique fondé sur une obséquiosité à l'égard des autorités, considérant que cette conception scolastique avait ruiné la médecine pendant des siècles ; voir D. Mugnai Carrara, «Epistemological Problems in Giovanni Mainardi’s Commentary on Galen's Ars Parva ", dans éd. A. Grafton et N. Siraisi, Natural Particulars : Nature and the Disciplines in Renaissance Europe (Cambridge [Mass.], Londres : MIT Press, 1999), p. 251-273. Pour se faire une idée de l'identité et du milieu socio-culturel des auditeurs de Brasavola, voir V. Nutton, « « Medicine, Diplomacy and Finance ».

35. V. Nutton, « Hellenism Postponed », p. 167. À ce sujet, il écrit : « Leoniceno's attack on Pliny, Valla and Benedetti's anatomy, Manardi's discussions of botany and diseases, all take as their starting point single Greek words ».

36. Galien, CRMA I 15 G (Kühn XV, 446, 5-7 = G. Helmreich, CMG V 9, 1 (Leipzig :

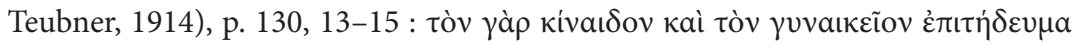

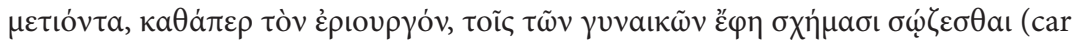
[il expliquait que] l'inverti et celui qui s'adonne à une activité féminine comme le lainier, étaient sauvés suivant le schéma des femmes).

37. De fait, en I 17 G (= sectio XVIII), les manuscrits qui offrent la leçon toĩ $\lambda$ oıtroĩ $\zeta \omega \mu$ oị sont l'Athous Iviron 184, le Vaticanus Reginensis gr. 173, le Marcianus App. 
Gr. V, 5 et le Trivultianus gr. 685 : c'est la leçon qu'il faut retenir. En I 22 G (= sectio XXIII, p. 56, col. a, 1. 58), Brasavola fait remarquer qu'un manuscrit consulté par

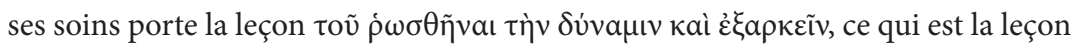
de L (Laurentianus gr. 75, 5) et de ses descendants (Marcianus gr. 281 et Parisinus gr. 2165).

38. Pour d'autres discussions sur le texte de l'Aldine, voir p. 33, col. b, 1. 62; p. 46, col. a, 1.56 sq. ; p. 58, col. b, 1. 54 sq. ; p. 93, col. a, 1. 27 sq.

39. Sur ce point capital, voir D. Mugnai Carrara, « Nicolò Leoniceno e Giovanni Mainardi ».

40. Galien, en expliquant « Hippocrate par Hippocrate », héritait déjà de la philologie

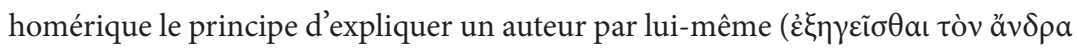

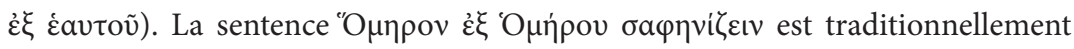
attribuée à Aristarque de Samothrace, bibliothécaire du Musée d'Alexandrie et commentateur d'Homère. Sur ce précepte exégétique, voir C. Schäublin, « Homerum ex Homero ", Museum Helveticum 34 (1977), p. 221-227.

41. Medici Romani Nicolai Leoniceni discipuli Antisophista (Bâle, 1532), 151 C.

42. Voir G. Helmreich, CMG V 9, 1, p. 148, 13-15.

43. Lege secundum de temperamentis librum prope finem. Methodus habetur inueniendi, quinam hominum sit temperatissimus, et deinceps quae sit singularum corporis partium temperatura. In tertio uero libro medicamentorum temperamenta ostendit. potissima tamen illius libri intentio est de homine loqui. De inanimatis tamen et praedicto libro agit et per omnes libros de simpl. medic. facul. tamen unus est particularis locus, in quo uniuersaliter edocemur quomodo simplicium temperaturae cognoscantur, lib. 5. cap. ulti. (p. 74, col. b, 1. 16-24).

44. I 17 H (= sectio XVIII, p. 25, col. b, 1. 61-63) : Circumfertur liber Galeno adscriptus de ptisana, qui etsi Galeni non sit, tamen est alicuius antiqui diligentis et probi.

45. Scito Galenum in hoc libro variis in locis de his mentionem fecisse, qui nimis implebant et nimis tenui victu reficiebant aegros, primo sit mentio superius sectione .13. et sectione .25. adde in secundo libro de inedia sectione .13. sermonem haberi, et sectione .15. et .34. et .40.43.44.47.48.54. Et tertio libro sectione .17.18. et quarto libro sectione .5. et sectione.13. de his qui quinta, vel.7. die recepturi sunt coctionem, et sectione.18. et 44 .

46. En commentant l'adjectif verbal $\beta \lambda \eta \tau$ ó $\varsigma$ du texte d'Hippocrate en I $34 \mathrm{H}$ (sectio XXXV, p. 81, col. a, 1. 64), Brasavola cite l'exemple du camp d’Agésipolis qui fut foudroyé (Helléniques IV, 7, 7): Habetur, et apud Xenophontem in quarto rerum graecarum, de fulminis ictu in castris. 
47. Sur ce point, voir V. Nutton, « The Rise of Medical Humanism », p. 17.

48. Sa notice sur le poète comique Eupolis est très fouillée, voir I $4 \mathrm{H}$ (sectio V, p. 8, col. b, 1. 58- p. 9, col. a, 1. 22).

49. Le texte de la Souda est cité en grec I 24 G (sectio XXV, p. 59, col. a, 1. 14-19).

50. Sur le garum, voir R. I. Curtis, Garum and Salsamenta: Production and Commerce in Materia Medica (Leyde, New York, Cologne : Brill, 1991).

51. Garum sociorum, garum liculmen, garum occultum, garum sanguiculum, garum nigrum, garum Hispanum, garum primum et garum fecosum.

52. Alias in errore fui, putans vocatum a nobis cauiarum esse garum antiquorum, cum tamen non sit, nam garus ex interaneis sit, cauiarum vero ex piscium ouis (...) sit uero cauiarum ex cuiuslibet piscis ouo, et coqui illustrissimi principis nostri Herculis Ferrariae Ducis praestantius cauiarum parant, quam dici possit (p. 43, col. b, 1. 2937).

53. Sur l'anti-arabisme à la Renaissance, voir $\mathrm{H}$. Schipperges, Ideologie und Historiographie des Arabismus, dans Sudhoffs Archiv. Beiheft 1 (Wiesbaden : Franz Steiner, 1961), p. 14-26 ; F. Klein-Franke, Die klassische Antike in der Tradition des Islam (Darmstadt : Wissenschaftliche Buchgesellschaft, 1980), p. 19-52 et P. E. Pormann, «La querelle des médecins arabistes et hellénistes et l'héritage oublié ».

54. In libros de ratione victus in morbis acutis, Hippocratis et Galieni commentaria et annotationes, p. 2, col. b, 1. 44-52 : Cur autem publicum morem praetermiserimus, qui est, ut is qui hanc sellam conscenderit, sub hoc munere auspicandi, quartum Auicennae canonem interpretetur : iam alias satisfactum est, Auicennam quidem summum uirum fuisse atque probum, verum non talem ut pro eo Hippocrates et Galenus deferendi sint. Propterea, quod nobis institutum est prosequemur, Hippocratis, scilicet et Galeni opera sectari, et illa publice interpretari, aliis omnibus posthabitis authoribus et potissimum Mauritanis.

55. Étonnamment, Brasavola semble ignorer que le texte a d'abord été traduit du grec à l'arabe avant d'être traduit en latin.

56. Abstinere non possum, quin compositionem quandam gari referam Galeno attributam, in librum etiam eidem authori attributo tertio de medic. facile parandis, quam compositionem cur garum uocetur, ignoramus, quoniam neque piscem garum, neque alterum piscem recepit, imo utitur abstrusis ponderum nominibus, est potius Araba quaedam compositio, quam a Graeco aliquo confecta, inscriptionem habet, confectio gari Ioachi Martyropolitae... (I 17 G = XVIII, p. 43, col. a, 1. 29-35).

57. Profecto si haec conditura vel Galeni vel alicuius probi viri fuisset, totis viribus insudassem ut multa in illa obscura interpretarer. cum vero nullius sit digni fide 
authoris neque sit antiquorum garus, qui ex piscium interaneis, et sale fiebat, in his exponendis quae obscura sunt non immorabimur (I 17 G = XVIII, p. 43, col. a, 1. 58-62).

58. A. M. Brasavola, Examen omnium simplicium medicamentorum (Lyon, 1537), p. 5.

59. Sur les fièvres au XVI ${ }^{\text {e }}$ siècle, voir I. M. Lonie, « Fever Pathology in the Sixteenth Century : Tradition and Innovation", dans éd. W. F. Bynum et V. Nutton, Theories of Fever from Antiquity to the Enlightenment, dans Medical History, Supplement 1 (1981), p. 19-44.

60. Sur ce point, voir N. Siraisi, « The Changing Fortune of a Traditional Text : Goals and Strategies in Sixteenth-Century Latin Editions of the Canon of Avicenna ", dans éd. A Wear, K. French et I. M. Lonie, The Medical Renaissance of the Sixteenth Century, p. 16-41, ici p. 21.

61. N. G. Siraisi, Avicenna in Renaissance Italy: The Canon and Medical Teaching in Italian Universities after 1500 (Princeton : Princeton University Press, 1987), ici p. $88-89$.

62. Voir N. Siraisi, « The Changing Fortune of a Traditional Text », p. 36-38.

63. Voir D. Mugnai Carrara, «Le epistole prefatorie sull'ordine dei libri di Galeno di Giovan Battista Da Monte : esigenze di metodo e dilemmi editoriali ", dans éd. V. Fera et A. Guida, Vetustatis Indagator. Scritti offerti a Filippo Di Benedetto (Messine : Centro di Studi Umanistici, 1999), p. 207-234, ici p. 223.

64. Voir G. B. Da Monte, dans Galeni omnia quae extant opera ex secunda Iunatrum editione [...], Venise, 1541, f. 4v-5r (cité par D. Mugnai Carrara, « Le epistole prefatorie ", p. 223, n. 39) : Atque hac ego occasione Avicennam commotum crediderim, virum inter Arabas omnes acerrimi ingenii, ut omnia Galeni in medica arte monumenta, sparsim in variis libris disseminata, in unum veluti corpus redigeret, eodemque compositivo ordine opus illud suum 'Canonum' appellatum, quinque magnis libris distinctum compilaret, cui si ultimam manum imposuisset (neque enim imposuit morte praeventus) feliciorique lingua pro rei dignitate elocutus esset, haberemus fortasse quem non Galeni tantum interpretem, sed exornatorem quoque vocitaremus.

65. Avicennae Liber Canonis, De Medicinis Cordialibus, et Cantica ... (Venise, 1555). Sur ce Benedetto Ricio, voir N. Siraisi, « The Changing Fortune of a Traditional Text », n. 53, p. 287.

66. Hoc in loco inter Galenum et Avicennam contradictio contingit, et Auicennae sectatores Galenum derisere (I $17 \mathrm{G}=$ XVIII, p. 44, col. a, 1. 36).

67. Voir I $42 \mathrm{G}=$ XLIII, p. 88 , col. b, 1. 48-66. 
68. E. Renan, Averroès et l'averroïsme (Paris : Maisonneuve et Larose, 2002), p. 282283.

69. Addit et exemplum Auerrois de duobus iuuenibus à Rasi propositum, qui in diebus canicularibus iter agebant, uterque causonem incidit, et domino fuit data potui aqua, qui sanatus est, seruo non fuit data et obiit (I 42 G = XLIII, p. 88, col. b, l. 67-p. 89, col. a, 1.2).

70. Brasavola signale toutefois que l'exemple des deux jeunes gens est en réalité tiré de Galien et non emprunté à Razi. Mais cela ne change rien pour lui à la question, car Galien interdisait l'eau avant la crise de la maladie ; voir I 42 G = XLIII, p. 89, col. a, 1. 28-37.

71. V. Nutton, dans "The Rise of Medical Humanism », p. 15, remarquait que contrairement à ses contemporains qui réprouvaient la polypharmacie arabe, Brasavola avait consacré un recueil aux sirops avec le même soin que pour les autres préparations pharmaceutiques : Examen omnium syruporum, quorum publicus usus est (Venise, 1538).

72. Sur ce point, voir K. H. Dannenfeldt, "The Renaissance Humanists and the Knowledge of Arabic », Studies in the Renaissance 2 (1955), p. 96-117 (repris dans éd. F. Sezgin, The Reception and Assimilation of Arabic Science in the Occident [Francfort-sur-le-Main : Institut für Geschichte der Arabisch-Islamischen Wissenschaften, 2005], tome v : p. 268-289) et G. Baader, "Medizinisches Reformdenken und Arabismus im Deutschland des 16. Jahrhunderts ", Sudhoffs Archiv 63 (1979), p. 261-296.

73. Sur Giorgio Merula, voir M. E. Consenza, Biographical and Bibliographical Dictionary of the Italian Humanists and of the World of Classical Scholarship in Italy, 1300-1800 (Boston : G. K. Hall, 1962), vol. 3, p. 2296-2302.

74. Sur cet érudit italien qui porte le même nom quele cousin de son père Zaccaria et qui est donc dit " minor », voir E. Bigi, s. v. "Barbaro Ermolao (Almoro) ", dans Dizionario Biografico degli Italiani (Rome : Istituto dell'Enciclopedia italiana, 1964), tome 6 : p. 96-99 ; M. E. Consenza, Biographical and Bibliographical Dictionary of the Italian Humanists and of the World of Classical Scholarship in Italy, 1300-1800 (Boston : G. K. Hall, 1962), vol.1 : p. 405- 412 ; V. Branca, "Lumanesimo veneziano alla fine del Quattrocento. Ermolao Barbaro e il suo circolo », dans éd. G. Arnaldi et M. Pastore Stocchi, Storia della cultura veneta III 1 (Vicenza : Neri Pozza, 1980), p. 123-175. 
75. Sur ce médecin d’origine bâloise, voir S. Fortuna, s. v. « Kopp Wilhelm », dans éd. F. J. Worstbrock, Deutscher Humanismus 1480-1520 : Verfasserlexikon, Bd. I, 2 (Berlin, New York : De Gruyter, 2006), col. 1309-1315.

76. Cette traduction et celle du Pronostic furent maintes fois rééditées, voir S. Fortuna, « Kopp Wilhelm », p. 1313.

77. Copus hic sensum facit, non quod cibus post duos dies augendus sit, sed quod iudicationis impetus magna ex parte duos dies non excedit. At nos dicimus neque unum excedere (I $24 \mathrm{H}$ = sectio XXV, p. 57, col. a, 1. 65-67). La traduction de Copp est effectivement : iudicationem cuius impetus magna ex parte duos dies non excedit. J'ai consulté cette traduction dans l'édition de Lyon de 1532 (chez Gryphe). Ce passage se trouve p. 174. Pour un autre contresens de la traduction de Copp relevé par Brasavola, voir I $40 \mathrm{H}=$ sectio XLI, p. 84, col. b, 1. 23-32.

78. Urbes uero Ferrariae propinquae, quae sunt prope montes, pisciculos habent variorum generum vere petrosos ex loco in quo habitant, ut Bononia, Florentia, Senae, Luca, Mutina, Regium Lepidi (I 26 G = sectio XXVII, p. 70, col. b, 1. 9-12).

79. Non mihi tempero, quin medici cuiusdam temeritatem adducam, qui Lucae prandente Pontifice .1541. in mense septembris, totis uiribus defendere conabatur, neminem ante Hippocratem de re medica quicquam scriptis mandasse (I $1 \mathrm{H}=$ sectio I, p. 3, col. a, 1. 50-53).

80. Ailleurs, il fait un commentaire ethnographique, quand, dans un développement sur les foies de volaille, il loue la façon qu'ont les Juifs de les préparer et rapporte que des Juifs de Ferrare lui firent don de ce mets fameux à chaque Noël : Hebraei ex pastillis e milii farina factis anseres pascunt, et mira iecuscula parant, accedunt quandoque ad duarum librarum pondus. a Ferrariensibus Hebraeis, singulis Christi natiuitatibus, his iecusculis donor (I $26 \mathrm{G}=$ sectio XXVII, p. 68, col. b, 1. 29-32).

81. Inter alpes, quae Italiam distinguunt a Gallia, in loco nuncupato Moriana a flumine coenoso ita denominato presbyterum vidimus cantorum, qui guttur habebat uiginti librarum pondere, ut omnes qui cum illustrissimo Hercule Duce Ferrariae in comitatu erant, iudicauere, ego autem ob duritiem et densitatem vigintiquinque librarum esse iudicabam (I 8 G = sectio IX, p. 14, col. a, 1. 27-32).

82. Nonnullos audiuimus per forum, et apud seplasiarios obmurmurantes, et stupida quadam admiratione affectos nos in hac aetate nondum (ut illis uidetur) matura, ausos esse, partem illam rei medicae facultatis in publicis scholis aggredi, quae praxis nuncupatur: cum superioribus annis illis solum munus hoc interpretandi hanc rei medicae partem committeretur, qui iam aetate ingrauescerent, et senio confecti essent (prol., p. 1, col. a, 1. 1-13). 
83. Sur ce recueil de curationes, voir C. Menini, " "Curationes A. M. Brasauoli" Contributo alla conoscenza dell'opera di A. M. Brasavola come medico pratico ", Rivista di storia delle scienze mediche e naturali 43 (1952), p. 255-261.

84. Nosque dicimus tres has partes esse necessarias, et unumquenque pro virili sua incumbere debere ut illas acquirat, et secundum illas recte agere sciat, cum in re medica nihil sit scire, et agere nescire (I $5 \mathrm{G}=$ sectio VI, p. 9, col. b, 1. 38-41).

85. Voir I 17 G (= sectio XVIII, p. 45, col. a, 1. 26-27).

86. Nos vero in praesentiarum delicatiori condimento utimur. Nam saccarum habemus apud antiquos ignotum, quo oxyzaccar conficimus ad hunc usum saccarumque est fere temperatum, neque flatuosas habet partes, uti mel (I $42 \mathrm{G}=$ sectio XLIII, p. 86, col. b, 1. 30-34).

87. Cette qualité de l’orge est énoncée dans le traité hippocratique du Régime, II, 40 (Littré VI, 536 = R. Joly et S. Byl, CMG I 2, 4, [Berlin : Akademie Verlag, 2003²]).

88. Voir Hippocrate, Ancienne médecine V, 4 (Littré I, 580-582 = J. Jouanna 124, 13-20).

89. Plusieurs traités consacrés à la ptisane sont publiés aux $\mathrm{XVI}^{\mathrm{e}}$ et $\mathrm{XVII}^{\mathrm{e}}$ siècles : Jean Lalamant, De ptisana sui temporis libellus (Genève, 1579) ; Giovanni Tomaso Minadoi, Philodicus sive Dialogus de ptisana, eiusque cremore non tantum pleuriticis sed et morbis aliis exhibenda (Venise, 1591) ; Alessandro Peccana, Commentarii della Scandella (Vérone, 1622). Sur la ptisane, voir mon article «Polemics on ptisan in Antiquity and beyond ", dans éd. A. Dalby, G. Grant, J. Wilkins, Plants and knowledge, Actes du Colloque international d'Exeter (9-10 mai 2008), à paraître.

90. Mirandum est de quibusdam recentibus, qui fateantur non haberi bonum hordeum, quoniam apud nos est brutorum cibus, neque ptisanam nostro tempore fieri posse, cum non adsint coqui, qui illam parare nouerint, de hordeo vero cum sit brutorum cibus, curam non exhibemus vel seminandi, vel colligendi, vel seruandi (I $17 \mathrm{H}=$ sectio XVIII, p. 26, col. a, 1. 8-14).

91. Cum nostrae mulieres domi meae ptisanam ut superius instituimus, egregie parent, et suavis sit, atque iocunda, imo loco vulgati farris ptisana pro minutali frequentissime utimur, et tota nostra familia, et alii externii plerunque et sapide illam comedunt, in diebusque quibus caro interdicitur, parant nonnumquam domi meae ex oleo, sale, porro, anetho et modico aceti, gustui meo absque aceto sapidior videtur, unde arbitrandum est illos fuisse infelicissimos medicos, qui coquos quonam modo conficeretur, edocere nesciuerint, cum nos una vice tantum mulierculam edocentes, illam instituerimus, ut nunquam erraret (I $26 \mathrm{H}$ = sectio XXVIII, p. 72, col. b, 1. 20-29).

92. Nostro autem tempore per hunc incessum procedere non possunt medici in morbis acutis, quoniam rarissimis placet ptisanae cremor, uel ptisana ipsa, sed cogimur fere 
uelimus nolimus hordei aquam praebere (...). his uero quibus ptisana non displicet, ptisanam damus, et ptisanae cremorem, tamen neque despicienda sunt quae nos exhibemus (I 18 H, sectio XIX, p. 45, col. b, 1. 49-57).

93. Si vero constaret crisim fuisse perfectam, procul dubio cibus statim exhibendus erat, et copiosior, et alterius generis, quam ptisana, nunc ptisanae loco, panem lotum praebemus, in seminum peponum expresso decoctum, aliaque ejusdem generis, cum Ferrarienses ptisanae assueti non sint, cum tamen probe confecta gustui sapiat. Ptisanae cremorem magis odio habeo quam ptisanam totam (I $24 \mathrm{H}=$ sectio XXV, p. 57, col. a, 1. 56-62).

94. Ces difficultés auxquelles se heurte Brasavola pour appliquer à la lettre le régime des anciens peuvent rappeler de semblables inadéquations exprimées, à la génération suivante, par Girolamo Mercuriale (1530-1606). Mercuriale, dans son De arte gymnastica, défend, en bon disciple de Galien, la gymnastique comme une pratique salutaire à la régulation des humeurs. Pourtant il souligne que la lutte et la gymnastique acrobatique des anciens ne sont pratiquées à son époque que par les couches sociales populaires et paysannes ou encore que certaines prescriptions galéniques sont inadaptées aux femmes ou aux membres du clergé ; voir N. G. Siraisi, "History, Antiquarianism and Medicine: The Case of Girolamo Mercuriale », Journal of the History of Ideas 64.2 (2003), p. 231-251, ici p. 244-245.

95. M. Brudus Lusitanus, Liber de ratione victus in singulis febribus secundum Hippocratem ad Anglos (Venise, 1544). Sur ce texte et cet auteur, voir V. Nutton, " "A Diet for Barbarians" : Introducing Renaissance Medicine to Tudor England », dans éd. A. Grafton et N. Siraisi, Natural Particulars : Nature and the Disciplines in Renaissance Europe, p. 275-293.

96. Galien s'en prend à plusieurs reprises dans son commentaire au régime de Pétronas. Pétronas (ou Pétron) d'Égine est un médecin de l'époque d'Hippocrate. Sur Pétronas et sa doxographie, voir Anonyme de Londres, XX, 1-24 (éd. H. Diels [Berlin : Reimer, 1893]) ; Celse, De Medicina III, 9 (éd. et tr. angl. W. G. Spencer [Londres: William Heinemann LTD-Harvard University Press, 1948], t. I : p. 268-271) ; Galien, De optima secta ad Thrasybulum (Kühn I, 144); Galien, De l'expérience médicale, (tr. fr. de Pierre Pellegrin, dans Traités philosophiques et logiques [Paris : GF Flammarion, 1998], p. 159, 160 et 182) ; enfin K. Deichgräber, s. v. « Petronas », R. E. XIX, 1, col. 1191-1192.

97. Sur ce point, voir A. Pietrobelli, « Le modèle des démonstrations géométriques dans la médecine de Galien », Bulletin de l’Association Guillaume Budé 2 (2009), p. 110-130. 
98. Voir O. Temkin, "Nutrition from Classical Antiquity to the Baroque », dans éd. I. Galdston, Human Nutrition Historic and Scientific Institute of Social and Historical Medicine (New York: International Universities Press, 1960) (repris dans O. Temkin, "On Second Thought" and Other Essays in the History of Medicine and Science (Baltimore, Londres : John Hopkins University Press, 2002), c. 10, p. 180-194) ; E. H. Ackerknecht, "The End of Greek Diet », Bulletin of the History of Medicine 45 (1971), p. 242-249 ; A. Drouard, « Perspectives historiques sur la notion de nutrition ", dans éd. F. Audoin-Rouzeau et F. Sabban, Un aliment sain dans un corps sain. Perspectives historiques, $\mathrm{II}^{\mathrm{e}}$ Colloque de l'Institut européen d'histoire et des cultures de l'alimentation (Tours : Presses Universitaires François Rabelais, 2007), p. 93-105.

99. Voir, par exemple, la doxographie de l'Anonyme de Londres.

100. S. Tissot, Essais sur les maladies des gens du monde (Lausanne, 1770), p. 16-27.

101. Voir W. Pagel, Paracelsus : An Introduction to Philosophical Medicine in the Era of the Renaissance (Bâle : Karger, 1958), p. 174.

102. Sur la découverte des processus de la digestion par Van Helmont, voir R. P. Multhauf, « J. B. Van Helmont's reformation of the Galenic doctrine of digestion », Bulletin of the History of Medicine 29 (1955), p. 154-163 ; W. Pagel, « Van Helmont's Idea on Gastric Digestion and the Gastric Acid », Bulletin of the History of Medicine 30 (1956), p. 524-536 ; W. Pagel, Joan Baptista Van Helmont. Reformer of Science and Medicine (Cambridge : Cambridge University Press, 1982), p. 129-140.

103. Sur ces régimes à l'intention des intellectuels, voir notamment $\mathrm{H}$. Schipperges, «Diätetik für den 'homo literatus'. Ein Historischer Beitrag zur Gesundheit der Gelehrten ", dans Semper attentus. Beiträge für Heinz Götze zum 8. August 1977 (Berlin, Heidelberg, New York : Springer, 1977), p. 308-316; W. F. Kümmel, « Der homo litteratus und die Kunst gesund zu leben ", dans éd. R. Schmitz et G. Keil, Humanismus und Medizin (Weinheim : Acta humaniora Verlag Chemie, 1984), p. $67-85$.

104. Voir V. Nutton, "Les exercices et la santé : Hiéronymus Mercurialis et la gymnastique médicale ", dans éd.J. Céard, M.-M. Fontaine et J.-C. Margolin, Le corps à la Renaissance, Actes du XXX $\mathrm{XX}^{\mathrm{e}}$ colloque de Tours 1987 (Paris : Aux amateurs de livres, 1990), p. 295-308. Sur L’Art de la gymnastique de Mercuriale, voir l'édition de J.-M. Agasse, Girolamo Mercuriale, L’Art de la gymnastique, Livre premier (Paris: Les Belles Lettres, 2006).

105. J. Trémolières, Les Boissons, physiologie, comportement, hygiène (Paris : Institut national d'hygiène, Ordre national des pharmaciens, 1957), Diététique 
140 ANTOINE PIETROBELLI

thérapeutique (Paris : G. Doin, 1958), Diététique et art de vivre : bien manger pour mieux vivre (Paris : Seghers, 1975), J. Trémolières et alii, Manuel élémentaire d'alimentation humaine (Paris : Éditions E. S. F., 1975). 